\title{
$\eta$-INVARIANTS AND THEIR ADIABATIC LIMITS
}

\author{
JEAN-MICHEL BISMUT AND JEFF CHEEGER
}

\section{Contents}

0 . Introduction

1. Algebraic preliminaries

2. The finite dimensional case; superconnections

(a) Odd dimensional base spaces

(b) Even dimensional base spaces

Appendix 1. Coupling to general superconnections

3. Auxiliary Grassman variables and auxiliary adiabatic limits

4. The infinite dimensional case; fibrations

(a) Elementary geometry of fibrations

(b) Odd dimensional base spaces

(c) Even dimensional base spaces

(d) The case in which the Dirac operator on the fiber is not invertible Appendix 2. Multifibrations

\section{INTRODUCTION}

Let $X$ be a compact manifold with nonempty boundary and let $P$ be a first order elliptic differential operator over $X$. In order for $P$ to have a well-defined index, a suitable boundary condition must be imposed.

In [APS], Atiyah-Patodi-Singer introduced a boundary condition which is particularly significant for applications. Their condition involves a first order selfadjoint operator, $A$, on $Y=\partial X$, which is naturally associated to $P$. Specifically, the boundary values of solutions, $h$, to $P h=0$, on $X$, are required to lie in the direct sum of the eigenspaces of $A$ corresponding to negative eigenvalues.

In this situation, an invariant of $A$ called the $\eta$-invariant, $\eta(A)$, enters into the index formula for $P$; see [APS]. Formally, $\eta(A)$, is equal to the number of positive eigenvalues of $A$ minus the number of negative eigenvalues. In actuality, $A$ has infinitely many eigenvalues of each sign and $\eta(A)$ must be defined by a regularization procedure; see $(0.3)$.

The invariant, $\eta(A)$, is not locally computable. That is, it cannot be obtained by integrating over $Y$, any differential form which is given in local coordinates

Received by the editors February 12, 1988 and, in revised form, July 22, 1988.

1980 Mathematics Subject Classification (1985 Revision). Primary 58-XX.

The second author was partially supported by NSF Grant No. DMS840596. 
by a canonical expression derived from the symbol of $A$. In fact, any such expression behaves multiplicatively when one passes to a finite covering of the underlying manifold. Examples show that the $\eta$-invariant does not always behave in this way.

As a consequence of its not being locally computable, the $\eta$-invariant is often difficult to compute explicitly.

Consider, on the other hand, a 1-parameter family of operators, $A_{t}$. Then $\eta\left(A_{t}\right)$ behaves smoothly, when reduced $\bmod Z$ and it turns out that the variation, $d \eta\left(A_{t}\right) / d t$, is locally computable in terms of $A_{t}, d A_{t} / d t$. This remarkable property of the $\eta$-invariant is closely related to its connection with index theory.

In the present paper, we study the limiting value of the $\eta$-invariant for Dirac operators, in situations in which the metric (or part of the metric) on the underlying manifold is multiplied by the factor $\varepsilon^{-1}$ and $\varepsilon \rightarrow 0$. We show that in the presence of an additional invertibility hypothesis, the $\eta$-invariant approaches a limiting value which is locally computable (or partly locally computable).

The operation of blowing up the metric is called passing to the adiabatic limit.

The additional invertibility hypothesis is described in more detail following $(0.4)$, below.

The original motivation for considering our problem comes from a paper of Witten, [W]. He considers a family of Dirac operators acting on an even dimensional manifold. The parameter space of his family is a circle and thus, the (odd dimensional) total space of his family is the total space of a fiber bundle over the circle. Witten gives an argument relating the holonomy of the determinant line bundle of the family to the limit of the $\eta$-invariant of the Dirac operator on the total space, when the metric on the circle is blown up.

Witten's result was proved rigorously in [BF] and [C3]. The emphasis in [BF] was on the superconnection formalism of Quillen, [Q], in relation to the proof given in [B1] of the local index theorem for families.

In [C3], two proofs are given, one based on Duhamel's principle and the second which exploited the connection with previous work on conical singularities; see $[\mathrm{C} 1, \mathrm{C} 2]$. For the case of the signature operator, an expression equivalent to that considered by Witten had arisen in [C2], when considering the variation of the $\eta$-invariant, for a space with isolated conical singularities. Finally, it was emphasized in [C3] that for a fibration of compact manifolds, $Z \rightarrow M \stackrel{\pi}{\rightarrow} B$, the $\eta$-invariant of the total space can be viewed as a renormalized difference of $\eta$-invariants, with coefficients in an infinite dimensional bundle whose fiber is a space of sections along the fiber, $Z$.

If, as above, we view the case of fibrations (discussed in §4) from the standpoint of infinite dimensional coefficient bundles, then there is a corresponding problem for finite dimensional coefficient bundles. This is treated in $\S \S 2$ and 3 . The analogy between the above two problems extends the analogy between the 
local index theorem for Dirac operators coupled to superconnections and (the second proof of) the local index theorem for families; [B1].

For the remainder of this introduction, we will restrict our attention to the case of fibrations and we will assume that the dimension of the base is odd. For obvious reasons, the statements of our results are slightly different in the cases $\operatorname{dim} B$ odd and $\operatorname{dim} B$ even, but the discussions of these two cases run parallel to one another.

Let $Z^{2 l} \rightarrow M^{2(k+l)-1} \stackrel{\pi}{\rightarrow} B^{2 k-1}$ be a fibration of compact oriented spin manifolds. We assume that the metric on $M$ is of the form

$$
g^{M}=\pi^{*}\left(g^{B}\right)+g^{Z}
$$

where $g^{B}$ is the metric on $B$ and $g^{Z}$ annihilates the orthogonal complement of the fibers. Let $\xi$ be a Hermitian vector bundle over $M$ with unitary connection and $\nabla^{\xi}$ and curvature $L^{\xi}$.

We denote by $D_{\varepsilon}^{M}$ the Dirac operator with coefficients in $\xi$ for the metric

$$
g_{\varepsilon}^{M}=\varepsilon^{-1} \pi^{*}\left(g^{B}\right)+g^{Z} .
$$

By definition the reduced $\eta$-invariant of $D_{\varepsilon}^{M}$ is

$$
\bar{\eta}\left(D_{\varepsilon}^{M}\right)=\frac{1}{2}\left[\operatorname{dim} \operatorname{ker} D_{\varepsilon}^{M}+\frac{1}{\Gamma(1 / 2)} \int_{0}^{\infty} t^{-1 / 2} \operatorname{tr}\left(D_{\varepsilon}^{M} e^{-\left(D_{\varepsilon}^{1 /}\right)^{2} t}\right) d t\right] .
$$

(The fact that the integral is convergent at $t=0$ is nontrivial; see [Gil], [BF], and $\S 3$ below).

Let $R^{B}$ be the curvature of $B$ and let $\widehat{A}\left(R^{B} / 2 \pi\right)$ be the differential form representing the $\hat{A}$-genus of $B$, which is obtained from the Chern-Weil homomorphism;

$$
\widehat{A}\left(R^{B} / 2 \pi\right)=\operatorname{det}\left[\left[\frac{R^{B} / 4 \pi}{\sin R^{B} / 4 \pi}\right]^{1 / 2}\right]
$$

We now assume that the Dirac operator along the fibers, $D^{Z}$, with coefficients in $\xi$, is invertible for all fibers $Z$ (in $\S 4$, we briefly describe how the discussion can be modified if this fundamental assumption does not hold). Our basic assertion is that there exists an explicit differential form, $\hat{\eta}$, on $B$, whose value at $p \in B$ depends only on (global) information on $Z_{p}=\pi^{-1}(p)$, and on the splitting of $T M$ into its horizontal and vertical subbundles, such that

$$
\lim _{\varepsilon \rightarrow 0} \bar{\eta}\left(D_{\varepsilon}^{M}\right)=\frac{1}{(2 \pi i)^{k}} \int_{B^{2 h-1}} \hat{A}\left(i R^{B}\right) \hat{\eta} .
$$

Since, a priori, $\lim _{\varepsilon \rightarrow 0} \bar{\eta}\left(D_{\varepsilon}^{M}\right)$ depends on global information on all of $M$, (0.5) represents a partial localization of the $\eta$-invariant (to the fibers). Let $R^{Z}$ denote the curvature of the subbundle, $T Z \subset T M$, which is tangent to the 
fibers, computed with respect to the connection obtained by projecting the Levi Civita connection for $g^{B}$ onto $T Z$. Then

$$
d \hat{\eta}=\frac{1}{(2 \pi i)^{l}} \int_{Z^{2 l}} \widehat{A}\left(i R^{Z}\right) \operatorname{tr}\left(e^{-L^{\xi}}\right),
$$

where $\int_{Z^{21}}$ denotes the operation of integration over the fiber.

The form occurring on the right-hand side of $(0.6)$ is a representative of the (normalized) Chern character of the index bundle of the family of Dirac operators, $D^{Z}$, over $B$. This representative was obtained in [B1], by an extension to infinite dimensions of the superconnection formalism of [Q]. Since we have assumed $D^{Z}$ is invertible for all $Z$, the above family is trivial. Thus, the form on the right-hand side of (0.6) must be exact and the form $\hat{\eta}$ makes it exact in a canonical way.

By referring to the formula for $\hat{\eta}$ (see (4.34)) one sees that $\hat{\eta}$ can be interpreted as a generalization to infinite dimensions of the form constructed in [Q], in order to transgress a Chern character form. Indeed, someone familiar with [Q] and with the Levi Civita superconnection of [B1], might be led to write down the formula for $\hat{\eta}$ by analogy with Quillen's formula.

However, in the proof of $(0.5)$, we do not set out with the express purpose of making the form on the right-hand side of $(0.6)$ exact. Rather, the form $\hat{\eta}$ appears when we manipulate the integral in (0.3). That $(0.6)$ should hold for a form obtained in this way can be perceived by noting that $(0.3)$ is itself part of a transgression formula. An understanding of the sense in which this is so is of importance in dealing with the $\eta$-invariant. It leads naturally to the "auxiliary Grassman variable" which played a crucial technical role in [B2, BF, BGS] and which will be used again in the present paper. It is also intimately related to the proof of the Atiyah-Patodi-Singer formula, by means of the cone construction; see [C2, Chou]. We will return to these points in $\S 3$ and in [BC2].

We will discuss applications of our work and its relation to that of other authors elsewhere (see in particular [ADS, BaM, M1, M2, S]). At this juncture we will point out just the following. The main results of this paper are stated for the $\eta$-invariant-the value at $s=0$ of the $\eta$-invariant, $\eta(s)$. However, our method of proof makes it immediately apparent that there is a corresponding result for the $\eta$-function itself. This more general result is actually necessary for the applications mentioned above (compare the argument of [C3, Appendix 3]). For some further comments pertaining to these applications, see Example A.2.7 below.

Last, we mention that the $\hat{\eta}$ forms arise naturally in the context of $L_{2}$-index theory on closed oriented pseudomanifolds with nonisolated conical singularities. There they appear in local formulas for the homology $\mathscr{L}$-classes (see [C2, $\S 9])$. This is explained further in [BC2] in which the $\hat{\eta}$-forms and the cone construction figure prominently. The main results of that paper and of the present one were announced in [BC1]. 


\section{Algebraic PRELIMINARIES}

(a) $Z_{2}$-graded algebras. A vector space, $Z$, is called $Z_{2}$-graded if it comes equipped with a direct sum decomposition $Z=Z_{0} \oplus Z_{1}$. This decomposition determines and is determined by an involution, $\rho$,

$$
\rho \mid Z_{j} \stackrel{\text { def }}{=}(-1)^{j} .
$$

A $Z_{2}$-graded vector space, $A=A_{0} \oplus A_{1}$, which is an algebra, is called a $Z_{2}$-graded algebra if

$$
A_{j} A_{j^{\prime}} \subset A_{j+j^{\prime}} .
$$

We will use subscripts on elements to denote their grading, e.g., $a_{j} \in A_{j}$. All such subscripts take values in $Z_{2}$.

If $Z$ is a graded vector space then $\operatorname{End}(Z)$ is naturally a $Z_{2}$-graded algebra, where $m_{2} \in \operatorname{End}_{j}(Z)$ if

$$
m_{j}\left(Z_{j^{\prime}}\right) \subset Z_{j+j^{\prime}} .
$$

A $Z_{2}$-graded representation of $A$ is a homomorphism, $\phi(A) \rightarrow \operatorname{End}(Z)$, for some $Z$, with $\phi\left(A_{j}\right) \subset \operatorname{End}_{j}(Z)$.

By definition, the left regular representation, $a \rightarrow L_{a}$, of $A$, is $Z_{2}$-graded by $A=A_{0} \oplus A_{1}$. But it may have another $Z_{2}$-grading, say $A=A_{0}^{\prime} \oplus A_{1}^{\prime}$ (which need not define a $Z_{2}$-graded algebra structure). Suppose there exists $\tau \in A$ such that $\tau^{2}=1$ and the \pm 1 -eigenspaces of $L_{\tau}$ define a $Z_{2}$-grading for the left regular representation of $A$. Then any representation, $\phi$, has a $Z_{2}$-grading by the \pm 1 -eigenspaces of $\phi(\tau)$.

The $Z_{2}$-graded algebra, $A$, is called supercommutative if all supercommutators,

$$
\left[a_{j}, a_{j^{\prime}}\right] \stackrel{\text { def }}{=} a_{j} a_{j^{\prime}}+(-1)^{1+j j^{\prime}} a_{j^{\prime}} a_{j}
$$

vanish.

If $A, \phi, \rho$ are as above ( $A$ not necessarily supercommutative) then

$$
\operatorname{tr}_{s}(a) \stackrel{\text { def }}{=} \operatorname{tr}(\rho \phi(a))
$$

defines a supertrace on $A$, that is a linear map whose kernel contains all supercommutators.

If $A, B$ are $Z_{2}$-graded algebras, their $Z_{2}$-graded tensor product is naturally isomorphic as a vector space to $A \otimes B$ (the elements of $A \hat{\otimes} B$ are written $a \hat{\otimes} b$ in place of $a \otimes b)$. The multiplication on $A \hat{\otimes} B$ satisfies

$$
\left(a \hat{\otimes} b_{j}\right)\left(c_{j^{\prime}} \hat{\otimes} d\right)=(-1)^{j j^{\prime}} a c_{j^{\prime}} \hat{\otimes} b_{j} d .
$$

If $A, B$ are supercommutative, so is $A \hat{\otimes} B$ with respect to its natural tensor product grading,

$$
A_{j} \hat{\otimes} B_{j^{\prime}} \subset(A \otimes B)_{j+j^{\prime}}
$$


determined by $\rho_{A} \otimes \rho_{B}$, where the involutions $\rho_{A}, \rho_{B}$ determine the gradings on $A, B$.

Let $\phi, \psi$ be representations of $A, B$ on $X, Y$. Let $\mathbb{C}^{2}=\mathbb{C} \oplus \mathbb{C}$ define the grading on $\mathbb{C}^{2}$ and let $J, K \in \mathrm{End}_{1}\left(\mathbb{C}^{2}\right)$ denote the involutions,

$$
\begin{aligned}
J & =\left[\begin{array}{cc}
0 & -i \\
i & 0
\end{array}\right], \\
K & =\left[\begin{array}{ll}
0 & 1 \\
1 & 0
\end{array}\right] .
\end{aligned}
$$

Note that $J K=-K J$. If the grading on $X \otimes Y \otimes \mathbb{C}^{2}$ is determined by the involution $1 \otimes 1 \otimes i J K$, then

$$
a_{j} \hat{\otimes} b_{j^{\prime}} \rightarrow \phi\left(a_{j}\right) \otimes \psi\left(b_{j}\right) \otimes J^{j} K^{j^{\prime}}
$$

defines a $Z_{2}$-graded representation of $A \hat{\otimes} B$.

Let $\phi, \psi$ be as above and let $\psi$ be $Z_{2}$-graded. Let $\rho$ be as in (1.1). Then

$$
a_{j} \hat{\otimes} b \rightarrow \phi\left(a_{j}\right) \otimes \rho^{j} \psi(b),
$$

defines a representation of $A \hat{\otimes} B$. If $\phi$ is also $Z_{2}$-graded, this representation is $Z_{2}$-graded for the tensor product grading on $X \otimes Y$.

Example 1.12. Let $L_{b}$ denote the image of $b \in B$ under the left regular representation. If this representation has a $Z_{2}$-grading such that for all $b, \rho$ satisfies

$$
L_{\rho(b)}=\rho L_{b}
$$

then by (1.11),

$$
A \hat{\otimes} B \simeq A \otimes B .
$$

If $\rho=L_{\tau}$, for some $\tau \in B$, then (1.13) holds.

Example 1.15. Let $\phi$ be a representation of $A$ and let $Y$ be a graded vector space. Putting $B=1, \psi(1)=\operatorname{Id}_{y}$ defines a representation of $A \simeq A \hat{\otimes} 1$ via (1.11).

(b) Clifford algebras. Let $\mathbb{C l}\left(V^{n}\right)$ denote the complex Clifford algebra of the real inner product space, $V^{n}$. Relative to an orthonormal basis, $\left\{e_{i}\right\}, \mathbb{C l}\left(V^{n}\right)$ is defined by the relations

$$
e_{i} e_{j}+e_{j} e_{i}=-2 \delta_{i j} \text {. }
$$

As a vector space $\mathbb{C l}\left(V^{n}\right)$ can be identified with the complex exterior algebra. The Clifford multiplication is then exterior multiplication minus interior multiplication. The elements $e_{(i)}=e_{i_{1}} \cdots e_{i_{j}},(i)=\left(i_{1}, \ldots, i_{j}\right), i_{1}<\cdots<i_{j}$, form a basis for $\mathbb{C} l\left(V^{n}\right)$. Put $|(i)|=j$. The subspaces $\mathbb{C} l_{0}\left(V^{n}\right), \mathbb{C l}_{1}\left(V^{n}\right)$ spanned by those $e_{(i)}$ with $|(i)|$ even (respectively odd) give $\mathbb{C} l\left(V^{n}\right)$ the structure of a $Z_{2}$-graded algebra and we have

$$
\mathbb{C} l\left(V^{n}\right) \simeq \mathbb{C} l\left(R^{1}\right) \hat{\otimes} \cdots \hat{\otimes} \mathbb{C} l\left(R^{1}\right)
$$


( $j$-factors). If $V$ is oriented, the element

$$
\tau= \begin{cases}i^{k} e_{1} \cdots e_{2 k-1}, & n=2 k-1, \\ i^{k} e_{1} \cdots e_{2 k}, & n=2 k\end{cases}
$$

is independent of the choice $\left\{e_{\alpha}\right\}$ and satisfies

$$
\tau^{2}=1
$$

For $n$ even, $\tau$ defines a grading for the left regular representation. As in (1.14), this gives the isomorphism

$$
\mathbb{C} l\left(V^{n} \oplus R^{2 j}\right) \simeq \mathbb{C} l\left(V^{n}\right) \otimes \mathbb{C l}\left(R^{2 j}\right) .
$$

For $n=2 k$, even, up to isomorphism, $\mathbb{C} l\left(V^{n}\right)$ has a unique irreducible module, $F$, which has dimension $2^{k}$ and is $Z_{2}$-graded (by $\phi(\tau)$ ). In fact, $\mathbb{C l}\left(V^{2 k}\right) \simeq \operatorname{End}(F)$. We will follow the standard convention and write $F_{+} \oplus F_{-}$ for $F_{0} \oplus F_{1}$. We write $\operatorname{tr}_{s}()$ for the supertrace on $\mathbb{C l}\left(V^{2 k}\right)$ defined as in (1.5).

If $n=2 k-1$ is odd $\mathbb{C} l\left(V^{n}\right)$ has two inequivalent irreducible modules, each of dimension $2^{k-1}$. For arbitrary $n$,

$$
e_{j} \rightarrow e_{j} \cdot e_{n+1},
$$

defines an isomorphism, $\mathbb{C} l\left(V^{n}\right) \simeq \mathbb{C l}_{0}\left(V^{n} \oplus R\right)$. Thus, for $n$ odd, we can regard $F$ for $V^{n} \oplus R$ as (inequivalent) modules over $\mathbb{C l}\left(V^{n}\right) \quad\left(\tau \rightarrow \pm \operatorname{Id}_{F^{ \pm}}\right)$. For $V^{2 k-1}$ oriented, the notation $\operatorname{tr}(a)$ refers to the representation $F_{+}$.

Lemma $1.22([\mathrm{G}])$. (1) If $n=2 k$ is even then

$$
\operatorname{tr}_{s}\left(e_{(i)}\right)= \begin{cases}0, & (i) \neq(1, \ldots, 2 k), \\ i^{-k} 2^{k}, & (i)=(1, \ldots, 2 k) .\end{cases}
$$

(2) If $n=2 k-1$ is odd and $|(i)| \geq 1$,

$$
\operatorname{tr}\left(e_{(i)}\right)= \begin{cases}0, & (i) \neq(1, \ldots, 2 k-1), \\ i^{-k} 2^{k-1}, & (i)=(1, \ldots, 2 k-1) .\end{cases}
$$

The irreducible modules of $\mathbb{C l}\left(V^{n} \oplus W^{m}\right)$ can be obtained from irreducible modules $F\left(V^{n}\right), F\left(W^{m}\right)$ for $\mathbb{C l}\left(V^{n}\right), \mathbb{C l}\left(W^{m}\right)$ as follows.

For $n, m$ odd, the module $F\left(V^{n}\right) \otimes F\left(W^{m}\right) \otimes \mathbb{C}^{2}$ of $(1.10)$ is irreducible.

For $m$ even, the module $F\left(V^{n}\right) \otimes F\left(W^{m}\right)$ of (1.11) is irreducible.

Finally, we note the effect of scaling the inner product $\langle$,$\rangle on V$. For any inner product, $\mathbb{C} l(V)$ coincides as a vector space with $\Lambda^{*}(V) \otimes \mathbb{C}$. Fix an inner product, $\langle$,$\rangle and let \mathbb{C} l_{\varepsilon}(V)$ denote $\Lambda^{*}(V)$ with Clifford multiplication, $O_{\varepsilon}$ coming from $\varepsilon^{-1}\langle$,$\rangle . Then the automorphism of \Lambda^{*} \otimes \mathbb{C}$ induced by $\varepsilon^{1 / 2} V \rightarrow V$, provides a natural isomorphism $\mathbb{C} l_{\varepsilon}(V) \simeq \mathbb{C} l(V)$. It also provides a natural isomorphism between the orthonormal frames $\left\{\varepsilon^{1 / 2} e_{i}\right\}$ for $\varepsilon^{-1}\langle$, and $\left\{e_{i}\right\}$ for $\langle$,$\rangle . Thus, although there is no canonical choice for the space$ 
$F(V)$ for $\langle$,$\rangle , any fixed choice also provides an irreducible module for \mathbb{C} l_{\varepsilon}(V)$ via the above isomorphism.

In the sequel, if $X$ is a riemannian spin manifold, we will always assume that the space of spinors has been chosen independent of the scaling parameter of the metric. As a consequence, the action of $\varepsilon^{1 / 2} e_{i} \in \mathbb{C} l_{\varepsilon}\left(T X_{x}\right)$ on $F_{x}$ is independent of $\varepsilon$ and the Dirac operator $D_{\varepsilon}$ corresponding to $\varepsilon^{-1}\langle$,$\rangle is$ $\varepsilon^{1 / 2} D$, where $D$ is the Dirac operator for $\langle$,$\rangle .$

\section{THE FINITE DIMENSIONAL CASE; SUPERCONNECTIONS}

In this section we calculate the limits of reduced $\eta$-invariants of certain Dirac operators coupled to superconnections, on closed oriented spin manifolds, when the metric, $g^{B}$, is blown up by a factor $\varepsilon^{-1}$. These $\eta$-invariants are shown to be independent of $\varepsilon$ when reduced $\bmod Z$. Under the appropriate invertibility hypothesis, the limit exists in $R$ and we identify it.

Our main purpose is to record that the formulas for $\lim _{\varepsilon \rightarrow 0} \hat{\eta}\left(D_{\varepsilon}\right)$ (see Theorems 2.28 and 2.43) are slight variants of analogous formulas of [B2] and can be proved by essentially the same arguments. We also introduce the rescaling technique of Getzler which plays a central role in $\S \S 3$ and 4 . In $\S 3$, the results of this section will be treated from a somewhat different viewpoint.

The discussions of parts (a) and (b) correspond to the cases of fibrations with even and odd dimensional fibers respectively. As a consequence, our assumptions will differ in parts (a) and (b).

(a) Odd dimensional base spaces. Let $B^{2 k-1}$ be a compact connected oriented riemannian manifold with metric $g^{B}$ and curvature $R^{B}$. We assume that $B^{2 k-1}$ has a spin structure and let $F^{B}$ denote a Hermitian bundle of spinors. The unitary connection on $F^{B}$, which is induced from the Levi Civita connection on $B$, will be denoted $\nabla^{B}$.

Let $\xi=\xi_{+} \oplus \xi_{-}$be a finite dimensional, $Z_{2}$-graded, Hermitian vector bundle over $B$, with orthogonal splitting. Let $\nabla^{\xi}$ be a unitary connection which preserves the splitting. The curvature of $\nabla^{\xi}$ is denoted by $L^{\xi}$. Often in what follows, we also use $\nabla^{\xi}$ to denote the induced exterior differentiation on forms with values in $\xi$, in which case we have $L^{\xi}=\left(\nabla^{\xi}\right)^{2}$. We give the bundle $F^{B} \otimes \xi$ the tensor product connection, $\nabla=\nabla^{B} \otimes 1+1 \otimes \nabla^{\xi}$.

The fiber, $\xi_{p}$, of $\xi$, plays the role of the space of smooth $L_{2}$-sections of the bundle of spinors of the fiber $Z$, of a fibration $Z^{2 l} \rightarrow M \rightarrow B^{2 k-1}$ (possibly tensored with a coefficient bundle). Since the grading on $\xi$ corresponds to the decomposition $F^{Z}=F_{+}^{Z} \oplus F_{-}^{Z}$, we write $\xi=\xi_{+} \oplus \xi_{-}$(instead of $\left.\xi_{0} \oplus \xi_{1}\right)$. We let $\rho$ be the involution, $\rho \mid \xi_{ \pm}= \pm 1$.

Keeping in mind (1.11), Example 1.15, and the discussion at the end of $\S 1$, we extend the natural representation of $\mathbb{C l}\left(B_{p}\right)$ on $F_{p}^{B}$ to a representation on $F_{p}^{B} \otimes \xi$ as in (1.11). 
If

$$
D^{\xi}=\sum f_{\alpha} \nabla_{f_{n}}
$$

denotes the Dirac operator on sections of $F^{B} \otimes \xi$ associated to this representation, then in matrix form,

$$
D^{\xi}=\left[\begin{array}{cc}
D^{\xi_{+}} & 0 \\
0 & -D^{\xi_{-}}
\end{array}\right]
$$

where $D^{\xi_{ \pm}}$denote the usual Dirac operators associated to the representations, $f_{\alpha} \rightarrow f_{\alpha} \otimes 1$ on $\xi_{ \pm}$. Thus, the $\eta$-invariant

$$
\eta\left(D^{\xi}\right)=\eta\left(D^{\xi_{+}}\right)-\eta\left(D^{\xi_{-}}\right)
$$

is a difference of $\eta$-invariants and the reduced $\eta$-invariant,

$$
\bar{\eta}\left(D^{\xi}\right)=\bar{\eta}\left(D^{\xi_{+}}\right)-\bar{\eta}\left(D^{\xi_{-}}\right)+\operatorname{dim} \operatorname{ker} D^{\xi_{-}}
$$

is a difference of reduced $\eta$-invariants $\bmod Z$.

We now consider the operator

$$
D^{\xi}+V=\left[\begin{array}{cc}
D^{\xi_{+}} & V_{-} \\
V_{+} & -D^{\xi_{-}}
\end{array}\right]
$$

where $V \in$ End_ $_{-}(\xi)$ is selfadjoint. The pair $\left(\nabla^{\xi}, V\right)$ determines a superconnection in the sense of [Q]. The operator $D^{\xi}+V$ is the analogue of the Dirac operator on the total space of a riemannian submersion, $Z^{2 l} \rightarrow M \stackrel{\pi}{\rightarrow} B^{2 k-1}$, for which the horizontal distribution is integrable. The role of the Dirac operator, $D^{Z}$, along the fibers is played by $V$. The finite dimensional analogue of the case of fibrations with nonintegrable horizontal distribution will be indicated in Appendix 1.

Note the relation

$$
f_{\alpha} V+V f_{\alpha}=0
$$

as a consequence of which $D^{\xi} V+V D^{\xi}$, the supercommutator of $D^{\xi}$ and $V$, is of order zero.

Theorem 2.7. Let $\left(\varepsilon^{1 / 2} D+V\right)$ be associated to the metric $\varepsilon^{-1} g$ and superconnection $\left(\nabla^{\xi}, V\right)$. Then $\bar{\eta}\left(\varepsilon^{1 / 2} D^{\xi}+V\right)$ is independent of $\varepsilon$, when reduced $\bmod Z$.

Proof. Since the reduced $\eta$-invariant of an operator does not change when the operator is multiplied by a constant, it suffices to show that

$$
\frac{d}{d s} \bar{\eta}(D+s V)=0 \text {. }
$$

By the standard formula for the variation of $\bar{\eta}$,

$$
\frac{d}{d s} \bar{\eta}(D+s V)=\frac{a_{k-1}}{\sqrt{\pi}},
$$


where

$$
\operatorname{tr}\left(V e^{-\left(D^{\xi}+V\right)^{2}}\right) \sim \sum_{j} a_{j} t^{j-(2 k-1) / 2} .
$$

Thus, our claim follows from

\section{Lemma 2.11.}

$$
\operatorname{tr}\left(V e^{-\left(D^{\xi}+V\right)^{2} t}\right)=0\left(t^{1 / 2}\right) .
$$

Proof. We use Getzler's method as adapted to the odd dimensional case in [BF]. We can pretend $B^{2 k-1}=R^{2 k-1}$, where the coordinates $\left\{y_{\alpha}\right\}$ of $R^{2 k-1}$ are normal coordinates. The operator $V e^{-(D+V)^{2} t}$ has a kernel in $\mathbb{C} l(T B) \hat{\otimes} \operatorname{End}(\xi)$ and it suffices to study its trace at the origin. In terms of local trivializations which are covariant constant at $y=0$, this kernel can be written in the form

$$
\sum f_{\alpha_{1}} \circ \cdots \circ f_{\alpha_{i}} \circ \hat{\otimes} k_{(\alpha)}(y, t) d y_{1} \cdots d y_{2 k-1},
$$

where $k_{(\alpha)}(y, t)=k_{(\alpha)}(0, y, t)$ and $\circ$ denotes Clifford multiplication.

Since $V \in$ End $_{-}(\xi)$, it is clear that $V e^{-(D+V)^{2} t}$ is odd with respect to the total grading on $\mathbb{C l}(T B) \hat{\otimes} \operatorname{End}(\xi)$. Thus, the parity of $k_{(\alpha)} \in \operatorname{End}(\xi)$ is opposite to that of $|(\alpha)|=i$. Odd elements of $\operatorname{End}(\xi)$ have trace 0 . So, by (1.24), the only term in (2.13) with nonzero trace corresponds to $(\alpha)=(1, \ldots, 2 k-1)$.

The proof will be concluded by examining the effect of Getzler's transformation, $G_{\delta^{1 / 2}}$, on the operator $\delta\left(D^{\xi}+V\right)^{2}$. By definition, this means that we conjugate this operator by the coordinate change,

$$
y_{\alpha} \rightarrow \delta^{1 / 2} y_{\alpha}
$$

make the replacement

$$
f_{\alpha} \rightarrow \delta^{-1 / 2} f_{\alpha}
$$

in the Clifford variables, and change the Clifford multiplication, $\circ$, to $\circ_{\delta}$, which satisfies

$$
\left(\delta^{-1 / 2} f_{\alpha}\right) \circ_{\delta}\left(\delta^{-1 / 2} f_{\beta}\right)+\left(\delta^{-1 / 2} f_{\alpha}\right) \circ_{\delta}\left(\delta^{-1 / 2} f_{\beta}\right)=-2 \delta_{\alpha \beta} .
$$

Thus, $\lim _{\delta \rightarrow 0} \circ_{\delta}=\wedge$, where $\wedge$ denotes exterior multiplication. By (2.6), we easily obtain

$$
\begin{gathered}
\lim _{\delta \rightarrow 0} G_{\delta^{1 / 2}}\left(\delta\left(D^{\xi} V+V D^{\xi}\right)\right)=0, \\
\lim _{\delta \rightarrow 0} G_{\delta^{1 / 2}}\left(\delta V^{2}\right)=0 .
\end{gathered}
$$

Thus (see $[\mathrm{G}]$ )

$$
\begin{aligned}
\lim _{\delta \rightarrow 0} G_{\delta^{1 / 2}}\left(\delta\left(D^{\xi}+V\right)^{2}\right) & =\lim _{\delta \rightarrow 0} G_{\delta^{1 / 2}}\left(\delta\left(D^{\xi}\right)^{2}+\delta\left(D^{\xi} V+V D^{\xi}\right)+\delta V^{2}\right) \\
& =\lim _{\delta \rightarrow 0} G_{\delta^{1 / 2}}\left(\delta\left(D^{\xi}\right)^{2}\right) \\
& =\mathscr{H}+L^{\xi}(0)
\end{aligned}
$$


where the operator $\mathscr{H}$, which acts on $\Lambda^{*}\left(T R^{2 k-1}\right)$, is a certain generalization of the Hermite operator and $L^{\xi}(0)$ is the curvature 2-form of $\xi$ at the origin in $R^{2 k-1}$. Although its detailed form is not needed in this argument, we note that the heat kernel of $\mathscr{H}+L^{\xi}(0)$ can be written down explicitly (compare [G]).

Clearly, the kernel of $G_{\delta^{1 / 2}}\left(V e^{-\delta\left(D^{\xi}+V\right)^{2} t}\right)$ is equal to

$$
\sum_{(\alpha)}\left(\delta^{-1 / 2} f_{\alpha_{1}}\right) \circ_{\delta} \cdots\left(\delta^{-1 / 2} f_{\alpha_{i}}\right) \circ_{\delta} \hat{\otimes} k_{(\alpha)}\left(\delta^{1 / 2} y, \delta t\right) \delta^{(2 k-1) / 2} d y_{1} \cdots d y_{2 k-1}
$$

Let $a_{(\alpha), j, \delta}$ denote the coefficient of $t^{j-(2 k-1) / 2} f_{\alpha_{1}} \circ_{\delta} \cdots f_{\alpha_{i}} \circ_{\delta} d y \cdots d y_{2 k-1}$ in the asymptotic expansion at $y=0$ of the kernel in (2.20). Then

$$
a_{(\alpha), j, \delta}=\delta^{j-|(\alpha)| / 2} a_{(\alpha), j, 1}
$$

The kernel of $V e^{-\left(\mathscr{H}+L^{\xi}\right) t}$ has an asymptotic expansion of the form

$$
\sum_{j,(\alpha)} a_{(\alpha), j, 0} t^{j-(2 k-1) / 2} f_{\alpha_{1}} \wedge \cdots f_{\alpha_{i}} \wedge d y_{1} \cdots d y_{2 k-1} .
$$

Since the coefficients in such expansions are determined locally from the differential operator, it follows from (2.19) that

$$
\lim _{\delta \rightarrow 0} \delta^{j-|(\alpha)| / 2} a_{(\alpha), j, 1}=\lim _{\delta \rightarrow 0} a_{(\alpha), j, \delta}=a_{(\alpha), j, 0} .
$$

Thus,

$$
\begin{gathered}
a_{(\alpha), j, 1}=0, \quad j<\frac{1}{2}|(\alpha)|, \\
a_{(\alpha),|(\alpha)| / 2,1}=a_{(\alpha),|(\alpha)| / 2,0}, \quad|(\alpha)| \text { even. }
\end{gathered}
$$

The coefficients, $a_{(\alpha), j, 0}$, can be read off from the known kernel of $V e^{-\left(\mathscr{H}+L^{\xi}(0)\right) t}$. But for our present purposes, it suffices to observe that since $j$ takes integer values, (2.24) implies that for $|(\alpha)|$ odd, the first nonvanishing coefficient is $a_{(\alpha),\{|(\alpha)|+1] / 2,1}$, the coefficient of $t^{|(\alpha)| / 2+1-k}$. Since only $|(\alpha)|=2 k-1$ effects the trace in (2.12), the lemma and Theorem 2.7 follow.

Now assume that $\xi_{+}, \xi_{-}$have the same dimension and that $V$ is invertible. Since $V$ is selfadjoint, $V^{2}$ is positive definite and the integrand in the definition of the form, $\hat{\eta}$, below is convergent at $\infty$. We put

$$
\hat{\eta} \stackrel{\text { def }}{=} \int_{0}^{\infty} \operatorname{tr}_{s}\left(V e^{-\left(\nabla^{\xi}+u^{1 / 2} V\right)^{2}}\right) \frac{d u}{2 u^{1 / 2}} .
$$

Here, as in the remainder of this section, the exponential is evaluated in $\Lambda^{*}(T B) \hat{\otimes}$ End $\xi$. The supertrace is given by

$$
\operatorname{tr}_{s}(\omega A)=\omega \operatorname{tr}_{s}(A),
$$

where $\omega \in \Lambda^{*}(B)$ and $A \in \operatorname{End}(V)$ (see $[\mathrm{BF},(1.24)]$ ). 
Theorem 2.28. Let $V$ be invertible. Then

$$
d \hat{\eta}=\operatorname{tr}_{s}\left(e^{-L^{\xi}}\right)
$$

where the right-hand side represents the (normalized) Chern character of $\xi_{+}-$ $\xi_{-} \in K^{0}\left(B^{2 k-1}\right)$. Moreover,

$$
\begin{aligned}
& \lim _{\varepsilon \rightarrow 0} \bar{\eta}\left(\varepsilon^{1 / 2} D^{\xi}+V\right)=\frac{1}{(2 \pi i)^{k}} \int_{B^{2 k-1}} \widehat{A}\left(i R^{B}\right) \hat{\eta}, \\
& \bar{\eta}\left(D^{\xi_{+}}\right)-\bar{\eta}\left(D^{\xi_{-}}\right) \equiv \frac{1}{(2 \pi i)^{k}} \int_{B^{2 k-1}} \widehat{A}\left(i R^{B}\right) \hat{\eta} \quad(\bmod Z) .
\end{aligned}
$$

Proof. Formula (2.29) is just the transgression formula of [Q]. (2.31) is an immediate consequence of Theorem 2.7 and (2.30).

To prove $(2.30)$ we first note that the smallest eigenvalue of $\left(\varepsilon^{1 / 2} D^{\xi}+V\right)^{2}$ is bounded below by some $\lambda_{0}>0$, for $\varepsilon$ sufficiently small. In fact,

$$
\left(\varepsilon^{1 / 2} D^{\xi}+V\right)^{2} \geq \varepsilon^{1 / 2}\left(D^{\xi} V+V D^{\xi}\right)+V^{2}
$$

and our claim clearly follows from the fact that $\left(D^{\xi} V+V D^{\xi}\right)$ has order zero. In particular, $\bar{\eta}\left(\varepsilon^{1 / 2} D^{\xi}+V\right)=\frac{1}{2} \eta\left(\varepsilon^{1 / 2} D^{\xi}+V\right)$, for $\varepsilon$ sufficiently small.

The remainder of the proof can be carried out by an argument analogous to that of [B2, Theorem 1.3]. The context there was even dimensional, but the same considerations which played a role in Theorem 2.7 allow us to use (1.24) in place of (1.23) and thus to transfer the argument to the odd dimensional case (see also Remark 2.33). We will not give further details since, in any event, the proof can be completed by an argument which is completely analogous to the one given in $\S 4$, where the infinite dimensional case corresponding to the present finite dimensional one is treated. A crucial feature of that argument is the use of an exponential transform based on an auxiliary Grassmann variable (compare also the discussion of $\S 3$ ).

Remark 2.33. Since $\xi_{+}$and $\xi_{-}$can be identified by $V, \bar{\eta}\left(D^{\xi}\right)$ can be regarded as the difference of the reduced $\eta$-invariants on the same bundle; see (2.2). From this observation and the local formula for the variation of the $\bar{\eta}$ one can deduce that the right-hand side of $(2.30)$ is equal to $\bar{\eta}\left(D^{\xi}\right) \bmod Z$. Thus, $\bmod Z,(2.30)$ follows from Theorem 2.7 .

(b) Even dimensional base spaces. Let $B^{2 k}$ be a closed connected oriented riemannian spin manifold. Let $\xi$ be a Hermitian vector bundle with unitary connection over $B^{2 k}$ and let $W$ be a selfadjoint endomorphism of $\xi$. The spin representation is graded by $\tau$. We consider the operator

$$
D+\tau W=\left[\begin{array}{cc}
W & D_{-} \\
D_{+} & -\bar{W}
\end{array}\right]
$$

where $D$ is the Dirac operator on $F^{B} \otimes \xi$.

Let $\varepsilon^{1 / 2} D+\tau W$ be associated to the metric $\varepsilon^{-1} g^{B}$. 
Theorem 2.35. $\bar{\eta}\left(\varepsilon^{1 / 2} D+\tau W\right)$ is independent of $\varepsilon$ when reduced $\bmod Z$. Moreover,

$$
\bar{\eta}\left(\varepsilon^{1 / 2} D+\tau W\right) \equiv \frac{1}{2} \text { Ind } D_{+} \quad(\bmod Z) .
$$

Proof. Since the asymptotic expansion, as $t \rightarrow 0$,

$$
\operatorname{tr}\left(\tau W e^{-(D+s \tau W)^{2} t}\right) \sim \sum a_{j} t^{j-k},
$$

contains only integral powers of $t$, it follows that

$$
\frac{d}{d s} \bar{\eta}(D+s \tau W) \equiv 0
$$

compare (2.9). For $s=0$, the operator $D e^{-D^{2} t}$ is odd. Hence $\operatorname{tr}\left(D e^{-D^{2} t}\right) \equiv 0$ which implies

$$
\begin{aligned}
\bar{\eta}(D) & =\frac{1}{2} \operatorname{dim} \operatorname{ker} D \\
& =\frac{1}{2} \operatorname{dim} \operatorname{ker} D_{+}+\frac{1}{2} \operatorname{dim} \operatorname{ker} D_{-} \\
& =\frac{1}{2} \operatorname{Ind} D_{+}+\operatorname{dim} \operatorname{ker} D_{-} . \text {q.e.d. }
\end{aligned}
$$

Now assume that $W$ is invertible. Let $\xi_{+}^{W}, \xi_{-}^{W}$ be the bundles whose fibers are the direct sums of the positive (respectively negative) eigenspaces of the invertible selfadjoint operator $W, \xi=\xi_{+}^{W} \otimes \xi_{-}^{W}$. Let $D^{\xi_{ \pm}^{W}}$ be the Dirac operators associated to the connections $\nabla^{\xi_{ \pm}^{W W}}$ obtained by projecting $\nabla^{\xi}$ onto $\xi_{ \pm}^{W}$. Of course, $\operatorname{Ind}\left(D_{+}^{\xi_{+}^{\prime \prime}}\right)$ and $\operatorname{Ind}\left(D_{+}^{\xi_{-}^{\prime \prime}}\right)$ are independent of the particular choice of connection and

$$
\frac{1}{2}\left[\operatorname{Ind}\left(D_{+}^{\xi_{+}^{\prime \prime}}\right)-\operatorname{Ind}\left(D_{+}^{\xi_{-}^{\prime \prime}}\right)\right] \equiv \frac{1}{2} \operatorname{Ind}\left(D_{+}^{\xi}\right) \quad(\bmod Z)
$$

To state our next result we need to introduce some formalism; see [Q, §5]. We let $\underline{\mathbb{C l}}\left(R^{1}\right)$ be the Clifford algebra on $R^{1}$ associated to the negative of the usual bilinear form. Thus, $\underline{\mathbb{C} l}\left(R^{1}\right)$ has a single generator, $\sigma$, with $\sigma^{2}=1$. We form the graded tensor product, $\Lambda^{*}(T B) \hat{\otimes} \underline{\mathbb{C} l}\left(R^{1}\right) \otimes$ End $\xi$ and put

$$
\operatorname{tr}_{\sigma}(\omega \hat{\otimes}(a \sigma+b) \otimes B) \stackrel{\text { def }}{=} \omega \operatorname{tr}(a B)
$$

and

$$
\operatorname{tr}_{\sigma}^{\text {even }}(\omega \hat{\otimes}(a \sigma+b) \otimes B)=\omega^{\text {even }} \operatorname{tr}(a B) .
$$

Theorem 2.43. If $W$ is invertible for each fiber, the form

$$
\hat{\eta}=\int_{0}^{\infty} \operatorname{tr}_{\sigma}^{\text {even }}\left(\sigma W e^{-\left(\nabla^{\xi}+u^{1 / 2} \sigma W\right)^{2}}\right) \frac{d u}{2 u^{1 / 2}}
$$

is closed and represents the (normalized) Chern character of $\xi_{+}^{W}-\xi_{-}^{W} \in K^{0}(B)$. Moreover,

$$
\begin{aligned}
\lim _{\varepsilon \rightarrow 0} \bar{\eta}\left(\varepsilon^{1 / 2} D+\tau W\right) & =\frac{1}{\sqrt{\pi}} \frac{1}{(2 \pi i)^{k}} \int_{B^{2 k}} \widehat{A}\left(i R^{B}\right) \hat{\eta} \\
& =\frac{1}{2}\left[\operatorname{Ind}\left(D_{+}^{\xi_{+}^{u}}\right)-\operatorname{Ind}\left(D_{+}^{\xi_{-}^{w}}\right)\right] .
\end{aligned}
$$


Proof. Note first that by (2.40) the second equality in (2.45) generalizes (2.39). The proof of (2.44) is analogous to that of (2.29), but now since $d \hat{\eta}$ is odd, we get $d \hat{\eta}=0$. Conjugation by $\tau$ shows that

$$
\operatorname{tr}\left(\tau W e^{-\left(\varepsilon^{1 / 2} D+\tau W\right)^{2} t}\right)=\operatorname{tr}\left(\tau W e^{-\left(\varepsilon^{1 / 2} D-\tau W\right)^{2} t}\right) .
$$

If we expand the exponential by Duhamel's principle we see that only odd powers of $\tau$ can effect the trace in (2.46). Similarly, we find that

$$
\operatorname{tr}\left(\left(\varepsilon^{1 / 2} D+\tau W\right) e^{-\left(\varepsilon^{1 / 2} D+\tau W\right)^{2} t}\right)=\operatorname{tr}_{s}\left(\left(-\varepsilon^{1 / 2} i D+W\right) e^{-\left(\varepsilon D^{2}-i \varepsilon^{1 / 2}[D, W]+W^{2}\right) t}\right) .
$$

Now the proof of the first equality in (2.45) can be carried out in the same way as that of (2.30).

To see the second inequality in $(2.45)$, let $W^{\prime}$ be the endomorphism

$$
W^{\prime}=W^{-1}\left(W^{2}\right)^{1 / 2}
$$

and put

$$
W_{a}=(1-a) W+a W^{\prime}, \quad 0 \leq a \leq 1 .
$$

Then $W_{a}$ is selfadjoint and invertible for all $s$. The even form $\theta$ on $B^{2 k} \times$ $[0,1]$, defined by

$$
\theta=\int_{0}^{\infty} \operatorname{tr}_{\sigma}^{\mathrm{even}}\left(\sigma W_{a} e^{-\left(\nabla^{\xi}+d a \partial_{a}+u^{1 / 2} \sigma W_{a}\right)^{2}}\right) \frac{d u}{2 u^{1 / 2}}
$$

is closed (see [Q]). By the standard argument, it follows that replacing $W$ by $W^{\prime}$ in

$$
\int_{0}^{\infty} \operatorname{tr}_{\sigma}^{\mathrm{even}}\left(\sigma W e^{-\left(\nabla^{\xi}+u^{1 / 2} \sigma W\right)^{2}}\right) \frac{d u}{2 u^{1 / 2}}
$$

changes this even form by an exact form. By a similar argument, we can replace the connection, $\nabla^{\xi}$, by a connection $\nabla^{W}$, for which $W^{\prime}$ is parallel. After these replacements have been made, performing the integral gives

$$
\frac{\sqrt{\pi}}{2} \operatorname{tr}_{s}\left(e^{-L^{\prime \prime}}\right)
$$

where $L^{W}=\left(\nabla^{W}\right)^{2}$ and the supertrace is with respect to the splitting $\xi=$ $\xi_{+}^{W} \oplus \xi_{-}^{W}$. Our claim now follows from the Atiyah-Singer index formula.

Remark 2.53. In [CoM] Connes and Moscovici have calculated the cyclic cocycles of Fredholm modules by transgression of Quillen's superconnection forms. Also, the results of Connes, [Co], who calculated the cyclic cocycles associated with Dirac operators were recovered in [B2] in the local superconnection formalism. Theorem 2.43 is closely related to all these results.

\section{APPENDIX 1. COUPLING TO GENERAL SUPERCONNECTIONS}

We indicate briefly how the preceeding results generalize when the Dirac operator is coupled to a superconnection involving forms of all degrees. We will 
restrict attention to the $\operatorname{dim} B^{2 k-1}$ odd (the case $\operatorname{dim} B^{2 k}$ is similar; compare parts (a) and (b) above).

A general superconnection can be written in the form $\nabla^{\xi}+V$ where

$$
V=\sum_{j=0}^{2 k-1} V_{j}
$$

is a sum of $j$-forms with values in $\operatorname{End}_{j+1}(\xi)$; see [Q]. Thus, we can view $V$ as odd element of $\Lambda^{*}(B) \hat{\otimes} \operatorname{End}(\xi)$. Let $\underline{V}$ denote the operator

$$
\underline{V}=\sum f_{\alpha_{1}} \circ \cdots \circ f_{\alpha_{j}} \circ V_{j}\left(f_{\alpha_{1}}, \ldots, f_{\alpha_{j}}\right) \text {, }
$$

where each $f_{\alpha_{i}}$ varies over an orthonormal basis of $B_{p}$. If we assume that $V_{j}$ takes values in selfadjoint (respectively skewadjoint) elements of End ${ }_{j+1}(\xi)$ for $j$ even (respectively $j$ odd) we can again form the selfadjoint operator, $D^{\xi}+\underline{V}$. In the context of fibrations considered in $\S 4$ there is a term corresponding to $j=2$ coming from the integrability tensor of the horizontal distribution. Note that $V_{1} \neq 0$ just corresponds to a change of connection on $\xi$.

If the metric on $B$ is multiplied by $\varepsilon^{-1}$, the expression in (A.1.2) gets replaced by

$$
\underline{V}(\varepsilon)=\sum \varepsilon^{j / 2} f_{\alpha_{1}} \circ \cdots \circ f_{\alpha_{j}} \circ V_{i}\left(f_{\alpha_{1}}, \ldots, f_{\alpha_{j}}\right)
$$

Put

$$
V(x)=\sum x^{1-j} V_{j}
$$

( $x$ will play the role of $u^{1 / 2}$ in (2.26)). Application of Getzler's transformation leads to a formula analogous to that of Theorem 2.28. However, some care must be taken in defining the form $\hat{\eta}$ because of the small time asymptotics. Put

$$
\gamma(s)=\int_{0}^{\infty} x^{2 s} \operatorname{tr}_{s}\left(\frac{d}{d x}(V(x))\left(e^{-\left(\nabla^{\xi}+V(x)\right)^{2}}\right) d x\right.
$$

for $s$ sufficiently large (note the negative exponents in (A.1.4)). This form has a meromorphic continuation to $\mathbb{C}$ with simple poles and the residue at $s=0$ is exact. If we define

$$
\hat{\eta}=\lim _{s \rightarrow 0} \gamma(s)-\frac{1}{s} \operatorname{Res}_{s=0} \gamma(s)
$$

then

$$
\lim _{\varepsilon \rightarrow 0} \hat{\eta}\left(\varepsilon^{1 / 2} D^{\xi}+\underline{V}(\varepsilon)\right)=\frac{1}{(2 \pi i)^{k}} \int_{B^{2 k-1}} \widehat{A}\left(i R^{B}\right) \hat{\eta} .
$$

Let $c_{0}$ denote the constant term in the asymptotic expansion as $x \rightarrow 0$ of $\operatorname{tr}_{s}\left(e^{-\left(\nabla^{\xi}+V(x)\right)^{2}}\right)$. Then
(A.1.8)
$d \hat{\eta}=c_{0}$. 
In our main result on fibrations (see 0.4 ) the form $\hat{\eta}$ has the same structure as the form just defined. In that case, however, the residue vanishes identically. This cancellation stems from the fact that analogues of $\nabla^{\xi}$ and of $V_{2}$ are not independent of each other. Both are functions of the geometry of the fibration.

\section{AUXILIARY GRaSSMANN VARIABles AND AUXILIARY ADIABatiC limits}

We indicated in $\S 2$ that Theorems 2.28 and 2.43 can be proved by transferring the argument of Theorem 1.3 of [B2], the proof of which depends on an "auxiliary Grassmann variable." Here we show how by introducing an auxiliary dimension and then blowing up the metric in its direction, the auxiliary Grassmann variable appears naturally. We find that the basic facts concerning the $\eta$-invariant in dimension $n$ are consequences of statements concerning the behavior of supertraces for an associated operator in dimension $n+1$, as the metric is blown up in the auxiliary direction. This viewpoint is related to the proof of the "holonomy theorem" in [BF].

These arguments can also be carried over quite directly to derive our results on $\eta$-invariants for fibrations, proved in $\S 4$. There, however, we will employ the auxiliary Grassmann variable in a manner analogous to that of [B2] (see also $[\mathrm{BF}])$.

In this section we will restrict our attention to the case of odd dimensional base spaces (the even dimensional case is similar; compare Remark 4.100).

To prove Theorem 2.28 , it suffices to show (the pointwise reflection)

$$
\begin{aligned}
& \operatorname{tr}\left(\varepsilon^{1 / 2} D^{\xi}+V\right) e^{-\left(\varepsilon^{1 / 2} D^{\xi}+V\right)^{2} t} \\
& \quad=\frac{\sqrt{\pi}}{(2 \pi i)^{k}}\left[\hat{A}\left(i R^{B}\right) \operatorname{tr}_{s}\left(V e^{-\left(\nabla^{\xi}+t^{1 / 2} V\right)^{2}}\right)\right]_{2 k-1}+(\varepsilon t)^{1 / 2} 0\left(1+t^{N}\right)
\end{aligned}
$$

for some $N$; compare [B2, $\mathrm{BF}, \mathrm{C} 3]$.

If we assume (3.1), we get

$$
\begin{aligned}
\lim _{\varepsilon \rightarrow 0} & \frac{1}{2 \Gamma(1 / 2)} \int_{0}^{T} t^{-1 / 2} \operatorname{tr}\left(\left(\varepsilon^{1 / 2} D^{\xi}+V\right) e^{-\left(\varepsilon^{1 / 2} D^{\xi}+V\right)^{2} t}\right) d t \\
& =\int_{B} \frac{1}{(2 \pi i)^{k}} \hat{A}\left(i R^{B}\right) \int_{0}^{T} \operatorname{tr}_{s}\left(V e^{-\left(\nabla^{\xi}+t^{1 / 2} V\right)^{2}}\right) \frac{d t}{2 t^{1 / 2}}
\end{aligned}
$$

Moreover, by the argument of [C3] (see (1.21)), we have

$$
\begin{aligned}
& \left|\frac{1}{2 \Gamma(1 / 2)} \int_{T}^{\infty} t^{-1 / 2} \operatorname{tr}\left(\varepsilon^{1 / 2} D^{\xi}+V\right) e^{-\left(\varepsilon^{1 / 2} D^{\xi}+V\right)^{2} t} d t\right| \\
& \quad \leq \text { const } \varepsilon^{-(2 k-1) / 2} e^{-\lambda_{0} T / 2} .
\end{aligned}
$$

By taking $T=2 \lambda_{0}^{-1}(2 k-1)|\log \varepsilon|$ (with $\lambda_{0}$ as in the proof given in $\S 2$ ) and using (3.1), (3.3), it follows that we can replace $T$ by $\infty$ in (3.2). This completes the proof of Theorem 2.28. 
Remark 3.4. Application of Getzler's transformation, $G_{(\varepsilon t)^{1 / 2}}$, shows that

$$
\lim _{\varepsilon \rightarrow 0} \operatorname{tr}\left(V e^{-\left(\varepsilon^{1 / 2} D+V\right)^{2} t}\right)=\frac{\sqrt{\pi}}{(2 \pi i)^{k}}\left[\widehat{A}\left(i R^{B}\right) \operatorname{tr}_{s}\left(V e^{-\left(\nabla^{\xi}+l^{1 / 2} V\right)^{2}}\right)\right]_{2 k-1} .
$$

Thus, (3.1) is actually equivalent to

$$
\lim _{\varepsilon \rightarrow 0} \operatorname{tr}\left(\varepsilon^{1 / 2} D^{\xi} e^{-\left(\varepsilon^{1 / 2} D^{\xi}+V\right)^{2} t}\right)=0
$$

(with suitable remainder estimates). However, (3.6) does not follow immediately from Getzler's method because of the presence of the operator $\varepsilon^{1 / 2} D^{\xi}$ in front of the exponential. Under rescaling, $\varepsilon^{1 / 2} D^{\xi}$ blows up like $\varepsilon^{-1 / 2}$.

Let $B^{2 k-1}$ be as in $\S 2$. Consider the manifold $S^{1} \times B$, viewed as $\left[\frac{1}{2}, \frac{3}{2}\right] \times B$ end points identified. Let $f(r)$ be a smooth positive function on $\left[\frac{1}{2}, \frac{3}{2}\right]$ with $f\left[\frac{3}{4}, \frac{5}{4}\right]=r$, such that

$$
g_{\delta}^{S^{1} \times B}=\delta^{-1} d r^{2}+f^{2}(r) g^{B}
$$

defines a smooth metric on $S^{1} \times B$. We extend $\xi=\xi_{+} \oplus \xi_{-}$and its connection trivially to $S^{1} \times B$. Also, we identify the bundle of spinors, $F^{S^{1} \times B^{2 k-1}}=$ $F_{+}^{S^{1} \times B} \oplus F_{-}^{S^{1} \times B}$, with the direct sum $F^{B} \oplus F^{B}$. Put $D=D^{B}, \mathscr{D}_{\delta}=D_{\delta}^{S^{1} \times B}$. Then

$$
\begin{aligned}
\mathscr{D}_{\delta} & =\left[\begin{array}{cc}
0 & -\delta^{1 / 2}\left(\partial_{r}+\frac{(2 k-1)}{2} \frac{f^{\prime}}{f}\right)+\frac{D}{f} \\
\delta^{1 / 2}\left(\partial_{r}+\frac{(2 k-1)}{2} \frac{f^{\prime}}{f}\right)+\frac{D}{f} & 0
\end{array}\right] \\
& =\delta^{1 / 2}\left(\partial_{r}+\frac{(2 k-1)}{2} \frac{f^{\prime}}{f}\right) C+\frac{\bar{D}}{f} .
\end{aligned}
$$

Here $C=-i J$ generates $\mathbb{C} l\left(R^{1}\right), \bar{D}=D K$, and $J, K$ are as in (1.8), (1.9).

$$
\begin{aligned}
& C^{2}=-1, \\
& C \bar{D}+\bar{D} C=0 .
\end{aligned}
$$

For clarity, in what follows we will work at the ordinary tensor product and use the operator $\mathscr{D} \rho$ (which is the equivalent of $D^{\xi}$ of $\S 2$ ). Thus, we will also assume that $C$ acts by $1 \otimes C$ and consider $\mathscr{D}_{\delta} \rho$.

On $\left[\frac{3}{4}, \frac{5}{4}\right]$, we put $r=1+u$ and do Getzler's transformation $G_{\delta^{1 / 2}}$ on the first variable $u \rightarrow \delta^{1 / 2} u, C \rightarrow \delta^{-1 / 2} C, \circ \rightarrow \circ_{\delta}$ where $\left(\delta^{-1 / 2} C\right) \circ_{\delta}\left(\delta^{-1 / 2} C\right)=1$, $\lim _{\delta \rightarrow 0}{ }^{\circ} \delta=\wedge$. We can easily find that

$$
\lim _{\delta \rightarrow 0} G_{\delta^{1 / 2}}\left(\left(\mathscr{D}_{\delta} \rho+V / f\right)\right)^{2}=-\partial_{u}^{2}-z(\bar{D} \rho+V)+(\bar{D} \rho+V)^{2}
$$


where $z^{2}=0$. By using $z \bar{D}=-\bar{D} z$, we get

$$
\begin{aligned}
e^{-\left[-\partial_{u}^{2}-z(\bar{D}+\rho V)+(\bar{D} \rho+V)^{2}\right] t} & =e^{-\left(-\partial_{u}^{2}\right) t} e^{z(\bar{D}+\rho V) t} e^{-(\bar{D} \rho+V)^{2} t} \\
& =e^{-\left(-\partial_{u}^{2}\right) t}[1+z(\bar{D}+\rho V) t] e^{-(\bar{D} \rho+V)^{2} t} .
\end{aligned}
$$

The operator $\left(\mathscr{D}_{\delta} \rho+V / f\right)^{2}$ has a heat kernel of the form

$$
\begin{aligned}
\sum_{(\kappa)} h_{(\kappa), \delta}(u, y, t) f_{\alpha_{1}} \cdots f_{\alpha_{j}} d u d y_{1} \cdots d y_{2 k-1} \\
\quad+\sum_{(\kappa)} k_{(\alpha), \delta}(u, y, t) C f_{\alpha_{1}} \cdots f_{\alpha_{j}} d u d y_{1} \cdots d y_{2 k-1}
\end{aligned}
$$

and the limiting operator in (3.11) has a heat kernel of the form

$$
\begin{aligned}
& \sum_{(\kappa)} h_{(\kappa), 0}(y, t) f_{\alpha_{1}} \cdots f_{\alpha_{j}} d u d y_{1} \cdots d y_{2 k-1} \\
& \quad+\sum_{(\kappa)} k_{(\alpha), 0}(y, t) z f_{\alpha_{1}} \cdots f_{\alpha_{j}} d u d y_{1} \cdots d y_{2 k-1} .
\end{aligned}
$$

Let $\operatorname{tr}_{s}()=\operatorname{tr}(\tau \rho)$ denote the supertrace for $g_{\delta}^{S^{1} \times B}$. By reasoning as in Lemma 2.11 , we find that the coefficients of the asymptotic expansion as $t \rightarrow 0$ (at $u=0)$ of $\operatorname{tr}_{s}\left(e^{-\left(\mathscr{U}_{\delta} \rho+V / f\right)^{2} t}\right)$ converge, as $\delta \rightarrow 0$, to those of $\operatorname{tr}_{\rho}\left(k_{(1, \ldots, 2 k-1), 0}(t)\right.$ (where $\left.\operatorname{tr}_{\rho}(A) \stackrel{\text { def }}{=} \operatorname{tr}(\rho A)\right)$.

It is clear from (3.12) that

$$
\operatorname{tr}_{\rho}\left(k_{(1,2, \ldots, 2 k-1), 0}(t)\right)=\frac{1}{2 \sqrt{\pi}} \operatorname{tr}\left(t^{1 / 2}(D \rho+V) e^{-(D \rho+V)^{2} t}\right) .
$$

On the other hand, the even dimensional version of the argument of Lemma 2.11 shows that for each $\delta$,

$$
\begin{aligned}
\lim _{t \rightarrow 0} \operatorname{tr}_{s}\left(e^{-\left(\mathscr{Z}_{s} \rho+V / f\right)^{2} t}\right) & =\frac{1}{(2 \pi i)^{k}}\left[\widehat{A}\left(i R^{S^{1} \times B}\right)\right]_{2 k}\left(\operatorname{dim} \xi_{+}-\operatorname{dim} \xi_{-}\right) \\
& =0
\end{aligned}
$$

(where one sees that the right-hand side vanishes either by direct computation or as a consequence of the metric's being conformally a product).

It follows from (3.15), (3.16) that

$$
\lim _{t \rightarrow 0} \operatorname{tr}\left(t^{1 / 2}(D \rho+V) e^{-(D \rho+V)^{2} t}\right)=0 .
$$

Since the left-hand side of (3.17) has an asymptotic expansion in integral powers of $t$, we obtain

$$
\operatorname{tr}\left((D \rho+V) e^{-(D \rho+V)^{2} t}\right)=0\left(t^{1 / 2}\right) .
$$

In particular, the integral on the left-hand side of (3.2) converges at $t=0$. Equivalently, it follows that the $\eta$-function, $\eta(s)$, for the operator $D^{\xi}+V$ is regular for $\operatorname{Re} s>-2$. 
The argument we have given should be compared to that of $[\mathrm{BF}]$ and the discussion of [C1, $\S \S 5$ and 6$]$.

Remark 3.19. If, in (3.7), we let $0<r<\infty$ and take $f(r)=r$, we obtain the metric cone on $B^{2 k-1}$, with the metric blown up in the horizontal direction (see [C2, Chou, BC2]). Put

$$
\operatorname{tr}_{s}\left(e^{-\left(\mathscr{Z}_{s} \rho+V / f\right)^{2} u}\right)=d r \wedge H(\delta, r, u)
$$

Then

$$
d \int_{a}^{r} H(\delta, s, u) d s=d r \wedge H(\delta, r, u) .
$$

By arguing as above, we find

$$
\lim _{\delta \rightarrow 0} H(\delta, s, u)=\frac{1}{2 \sqrt{\pi}} \frac{u^{1 / 2}}{s^{2}} \operatorname{tr}\left((D \rho+V) e^{-(D \rho+V)^{2} u / s^{2}}\right) .
$$

Thus, putting $u / s^{2}=t$, we get

$$
d\left[\frac{1}{\sqrt{\pi}} \int_{u / r^{2}}^{u / a^{2}} t^{-1 / 2} \operatorname{tr}\left((D \rho+V) e^{-(D \rho+V)^{2} t}\right) d t\right]=d r \wedge H(\delta, r, u) .
$$

By letting $u / a^{2} \rightarrow \infty, u / r^{2} \rightarrow 0, u \rightarrow 0$, we obtain the integral which defines the $\eta$-invariant and we see the sense in which this integral represents a transgression formula for the adiabatic limit of the supertrace of the heat kernel of a Dirac operator on the cone; see [BC2] for further discussion.

We now indicate how (3.1) can be proved in the context of the present discussion. We replace $\mathscr{D}_{\delta} \rho$ by $\varepsilon^{1 / 2} \mathscr{D}_{\delta} \rho$ in (3.11) and obtain

$$
\lim _{\delta \rightarrow 0} G_{\delta^{1 / 2}}\left(\left(\varepsilon^{1 / 2} \mathscr{D}_{\delta} \rho+V / f\right)\right)^{2}=-\varepsilon \partial_{u}^{2}-\varepsilon^{1 / 2} z\left(\varepsilon^{1 / 2} \bar{D} \rho+V\right)+\left(\varepsilon^{1 / 2} \bar{D} \rho+V\right)^{2}
$$

Then, at $u=0$, we get

$$
\lim _{\delta \rightarrow 0} \operatorname{tr}_{s}\left(e^{-\left(\varepsilon^{1 / 2} \mathscr{Z}_{\delta} \rho+V / f\right)^{2} t}\right)=\frac{1}{\sqrt{\pi}} \operatorname{tr}\left(t^{1 / 2}\left(\varepsilon^{1 / 2} D \rho+V\right) e^{-\left(\varepsilon^{1 / 2} D \rho+V\right)^{2} t}\right)
$$

(compare (3.15) and the discussion preceding it). Let

$$
\tilde{\nabla}^{\xi}=\nabla^{\xi}+d r \wedge \partial_{r}
$$

be the extension of $\nabla^{\xi}$ to $S^{1} \times B$ and let $R_{\delta}$ be the curvature tensor of $g^{S^{1} \times B}$. By applying $G_{(t t)^{1 / 2}}$ we find that

$$
\lim _{\varepsilon \rightarrow 0} \operatorname{tr}_{s}\left(e^{-\left(\varepsilon^{1 / 2} \mathscr{Z _ { s }} \rho+V / f\right)^{2} t}\right)=\frac{1}{(2 \pi i)^{k}}\left[\widehat{A}\left(i R_{\delta}\right) \operatorname{tr}_{s}\left(e^{-\left(\tilde{\nabla}^{\xi}+t^{1 / 2} V / f\right)^{2}}\right)\right]_{2 k} .
$$

As previously mentioned, for each $\delta, \widehat{A}\left(i R_{\delta}\right)$ coincides with $\pi^{*} \widehat{A}(i R)$ where $\pi: S^{1} \times B \rightarrow B$. Thus, the right-hand side of (3.27) is independent of $\delta$. Let us grant that the limit as $\varepsilon \rightarrow 0$, of the right-hand side of (3.25), is equal to the 
limit as $\delta \rightarrow 0$, of the right-hand side of (3.27). Then, using (3.26), we easily obtain (3.1) (modulo the error estimate).

The assertion that the expression in (3.25) is equal to $H$ amounts to the fact that we can interchange the order in which we take the limits $\varepsilon \rightarrow 0, \delta \rightarrow 0$. In the discussion above, this was justified by remarking that the asymptotic expansion depends continuously on the operator (as we converge to the limiting operator in (3.11)). A similar argument works in this case. The construction of the expansion which we now indicate, while not the simplest, would also work in the case of fibrations considered in $\S 4$.

We start with the metric $g_{\delta}^{S^{1} \times B}$ and coordinates $u=(r-1) / \delta$. Then we express $\varepsilon^{-1} g_{\delta}^{S^{1} \times B}$ in coordinates $u \rightarrow \varepsilon^{1 / 2} u, y \rightarrow \varepsilon^{1 / 2} y$. The estimates and arguments of [C3, $\S \S 3$ and 4], produce an asymptotic expansion in powers of $\varepsilon$ for $\operatorname{tr}_{s}\left(e^{-\left(\varepsilon^{1 / 2} \mathscr{Z}_{s} \rho+V / f\right)^{2} t}\right)$. A priori, this expansion contains singular terms, but these must vanish by the discussion above. One easily shows that the coefficients and remainder term depend smoothly on $\delta$. As $\delta \rightarrow 0$ the metric on $S^{1} \times B$ converges to a product metric on $R^{1} \times B$. The coefficients and remainder vanish in the limit, $\delta \rightarrow 0$, as a consequence of the orientation reversing isometry in the $u$ direction. By using the differentiability with respect to $\delta$ and changing back to the $r$ coordinate, we obtain the desired interchange of limits. The estimates on the remainder term also can be read off from the expansion.

Note also that in (3.27), we are using Getzler's transformation for fixed time $t$ (not just for small time).

\section{The infinite dimensional CASE; FIBRATIONS}

Let $Z \rightarrow M \stackrel{\pi}{\rightarrow} B$ be a fibration of closed oriented riemannian manifolds such that $\pi$ is a riemannian submersion. Thus, the metric on $M$ is of the form

$$
g^{M}=\pi^{*}\left(g^{B}\right)+g^{Z},
$$

where $g^{Z}$ annihilates the orthogonal complement of the tangent space to the fiber.

We will assume that the tangent bundle, $T B$, and the tangent bundle to the fibers, $T Z \subset T M$, both have spin structures. Then, using the splitting, $T M=T^{H} M \oplus T Z$, into horizontal and vertical subbundles, we obtain a spin structure on $T M$.

Let $\xi$ be a Hermitian vector bundle over $M$, with unitary connection, $\nabla^{\xi}$. Let $D_{\varepsilon}$ be the Dirac operator on $M$, with coefficients in $\xi$, associated to the metric

$$
g_{\varepsilon}^{M}=\varepsilon^{-1} \pi^{*}\left(g^{B}\right)+g^{Z} .
$$

The following was observed in $[\mathrm{BF}, \mathrm{C}]$.

Proposition 4.3. If $M$ is odd dimensional, $\lim _{\varepsilon \rightarrow 0} \bar{\eta}\left(D_{\varepsilon}\right)$ exists in $R / Z$.

In fact as $\varepsilon \rightarrow 0$, the Levi Civita connection, $\nabla^{L, \varepsilon}$, approaches a limiting connection $\nabla^{L .0}$ (see $(4.16)$ below). Then the existence of $\lim _{\varepsilon \rightarrow 0} \bar{\eta}\left(D_{\varepsilon}\right)$ 
follows from the fact that the variation of $\bar{\eta}\left(D_{\varepsilon}\right)$ coincides with the variation of the Chern-Simons type invariant which appears in the Atiyah-Patodi-Singer formula. The latter depends only on the connection.

If the Dirac operator, $D^{Z}$, is invertible for each fiber $Z$, it is easy to see that $D_{\varepsilon}$ is invertible for $\varepsilon$ sufficiently small. Hence, in this case, $\lim _{\varepsilon \rightarrow 0} \bar{\eta}\left(D_{\varepsilon}\right)$ exists in $R$ (not just in $R / Z$ ) and we will derive a formula for it. In part (d) below, we indicate how the assumption that $D^{Z}$ is always invertible can be relaxed.

The cases of odd and even dimensional base spaces are treated in parts (b) and (c) respectively. We will use the generalization to infinite dimensions, given in [B1], of the superconnection formalism of [Q].

(a) Elementary geometry of fibrations. We begin by recalling some results of [B1]. Let $Z \rightarrow M \stackrel{\pi}{\rightarrow} B$ be a fibration of smooth manifolds. The subbundle of $T M$ whose fibers are the subspaces of $T M$ tangent to the fibers, $Z$, will be denoted by $T Z$. A connection on $Z \rightarrow M \rightarrow B$ is a complementary subbundle $T^{H} M$, i.e., $T M=T^{H} M \oplus T Z$. Let $P^{H}, P^{Z}$ denote the projections on $T^{H} M$, $T Z$ relative to this splitting.

If $U$ is a locally defined vector field on $B$, we denote by $\widetilde{U}$ the unique locally defined section of $T^{H} M$ such that $\pi_{*}(\tilde{U})=U$. The integrability tensor (or curvature) of $T^{H} M$ is the 2-form, $\mathscr{R}$ on $T^{H} M$ with values in $T Z$ defined by

$$
\mathscr{R}(\widetilde{U}, \widetilde{V})=-[\widetilde{U}, \widetilde{V}]+[\widetilde{U, V}]=-P^{Z}[\widetilde{U}, \widetilde{V}]
$$

The value of $\mathscr{R}(\widetilde{U}, \widetilde{V})$ at $m \in M$ depends only $\widetilde{U}(m), \widetilde{V}(m)$.

Let $\nabla^{Z}$ be a smoothly varying family of connections on tangent bundles to the fibers. This family determines a connection (also denoted $\nabla^{Z}$ ) on $T Z$ by putting

$$
\nabla \stackrel{X}{\widetilde{U}} W=[\widetilde{U}, W]
$$

for any local horizontal lift, $\widetilde{U}$, and vertical field, $W$.

Suppose the fibers of $T Z$ are equipped with a smoothly varying family of inner products. If we apply the above procedure to the corresponding family of Levi Civita connections on the fibers, $Z$, we do not necessarily obtain an orthogonal connection. We can correct for this by defining $A_{W}: T M \rightarrow \operatorname{End}(T Z)$ by $A_{W} \equiv 0$ for $W$ vertical and

$$
L_{\widetilde{U}} g^{Z}\left(W_{1}, W_{2}\right)=g^{Z}\left(A_{\widetilde{U}}\left(W_{1}\right), W_{2}\right)
$$

for $\widetilde{U}$ a horizontal lift. Here $g^{Z}$ is as in (4.1), $L_{\widetilde{U}}$ denotes Lie derivative, and $W_{1}, W_{2}$ are vertical. Then

$$
\nabla_{\widetilde{U}}^{Z}+\frac{1}{2} A_{\widetilde{U}}=[\widetilde{U}, \cdot]+\frac{1}{2} A_{\widetilde{U}}
$$

preserves the inner products on $T Z$. 
Alternatively, let $g^{M}$ be any riemannian metric on $M$, which extends $g^{Z}$ and for which $T^{H} M \oplus T Z$ is an orthogonal splitting. If $\nabla^{L}$ denotes the Levi Civita connection of $g^{M}$, then

$$
\nabla \underset{\widetilde{U}}{Z}+\frac{1}{2} A_{\widetilde{U}}=P^{Z} \nabla \underset{\widetilde{U}}{L}
$$

Moreover,

$$
I_{\widetilde{U}^{\prime}}\left(W_{1}, W_{2}\right)=g^{M}\left(A_{\widetilde{U}}\left(W_{1}\right), W_{2}\right)
$$

is the second fundamental form of the fiber.

From now on, we assume that the metric $g^{M}$ is of the form (4.1). Let $\nabla^{B}$ denote the Levi Civita connection for $g^{B}$. Note that $\nabla^{B}$ does not change when $g^{B}$ is multiplied by a constant. We define a connection $\nabla$ on $T M$ by

$$
\nabla=\pi^{*}\left(\nabla^{B}\right) \oplus \nabla^{Z}
$$

and let $S$ denote the difference tensor,

$$
S=\nabla^{L}-\nabla
$$

Then, for $\widetilde{U}_{1}, \widetilde{U}_{2}$ horizontal lifts, $W_{1}, W_{2}$ vertical (and for \langle\rangle$=g^{M}$ ) we have

$$
\left\langle S\left(W_{1}\right) W_{2}, \widetilde{U}_{1}\right\rangle=-\left\langle S\left(W_{1}\right) \widetilde{U}_{1}, W_{2}\right\rangle=\frac{1}{2}\left\langle A_{\widetilde{U}_{1}}\left(W_{1}, W_{2}\right\rangle\right.
$$

$$
\left\langle S\left(\widetilde{U}_{1}\right) W_{1}, \widetilde{U}_{2}\right\rangle=\left\langle S\left(\widetilde{U}_{1}\right) \widetilde{U}_{2}, W_{1}\right\rangle=\left\langle S\left(W_{1}\right) \widetilde{U}_{1}, \widetilde{U}_{2}\right\rangle=\frac{1}{2}\left\langle\mathscr{R}\left(\widetilde{U}_{1}, \widetilde{U}_{2}\right), W_{1}\right\rangle .
$$

All other components of $\langle S(\cdot), \cdot\rangle$ vanish. Clearly, $\langle S(\cdot) \cdot, \cdot\rangle$ is independent of $g^{B}$. Also,

(i) if $V \in T M$ then $S(V): T^{H} M \rightarrow T Z$,

(ii) if $\widetilde{U}_{1}, \widetilde{U}_{2} \in T^{H} M$ then $S\left(\widetilde{U}_{1}\right) \widetilde{U}_{2} \in T Z$,

(iii) if $\widetilde{U} \in T^{H} M$ then $S(\widetilde{U}) \widetilde{U}=0$.

It follows easily from $(4.12),(4.13)$ if $\nabla^{L, \varepsilon}$ is the Levi Civita connection of $g_{\varepsilon}^{M}$ of (4.2) and $S^{\varepsilon}=\nabla^{L, \varepsilon}-\nabla$, then

$$
\begin{aligned}
& p^{H} S^{\varepsilon}=\varepsilon P^{H} S, \\
& P^{Z} S^{\varepsilon}=P^{Z} S
\end{aligned}
$$

and hence that the limit,

$$
\lim _{\varepsilon \rightarrow 0} \nabla^{L, \varepsilon}=\nabla^{L}-P^{H} S
$$

exists.

The following discussion may be helpful in understanding the analogy between the results of $\S \S 2$ and 3 and those of parts (b) and (c) of this section.

Let $\gamma$ be an arbitrary vector bundle over $M$. Associated to $\gamma$ is a vector bundle, $\tilde{\gamma}$, over $B$ whose (infinite dimensional) fiber, $\tilde{\gamma}_{p}$, is the space of 
smooth sections of $\gamma \mid Z_{p}$ (where $Z_{p}=\pi^{-1}(p)$ ). There is an obvious functorial isomorphism between the space of smooth sections of $\gamma$ and the space of sections of $\tilde{\gamma}$ which are smooth in the appropriate sense.

If $\gamma$ has a connection, $\nabla^{\gamma}$, then we define the associated connection, $\widetilde{\nabla}$ on $\tilde{\gamma}$ by

$$
\tilde{\nabla}_{U} \tilde{s}=\nabla \frac{\gamma}{\widetilde{U}} s
$$

where $\tilde{s}$ denotes the section $s$ of $\gamma$, regarded as a section of $\tilde{\gamma}$. Clearly, the curvature, $\widetilde{R}$, of $\widetilde{\nabla}$, is given by

$$
\widetilde{R}\left(U_{1}, U_{2}\right)=R^{\gamma}\left(\widetilde{U}_{1}, \widetilde{U}_{2}\right)-\nabla_{\mathscr{H}\left(\widetilde{U}_{1} \widetilde{U}_{2}\right)}^{\gamma} .
$$

If $\gamma$ has a Hermitian inner product $($,$) and the fibers have a smoothly vary-$ ing family of volume forms, $\omega^{Z}$, then (for $Z$ compact) we define a fiberwise inner product on $\tilde{\gamma}$ by

$$
\left(\tilde{s}_{1}, \tilde{s}_{2}\right)=\int_{Z_{p}}\left(s_{1}, s_{2}\right) \omega^{Z_{p}}
$$

Even if $\nabla^{\gamma}$ is unitary, $\widetilde{\nabla}$ need not preserve the inner product in (4.19). Let $\omega^{Z}$ be extended to $T M$ so that it annihilates horizontal vectors and put

$$
L_{\widetilde{U}} \omega^{Z} \mid Z=M_{\widetilde{U}} \omega^{Z}
$$

Then

$$
\widetilde{\nabla}_{U}^{u}=\widetilde{\nabla}_{U}-\frac{1}{2} M_{\widetilde{U}}
$$

is unitary on $\tilde{\gamma}$. If, as we now assume, $\omega^{Z}$ is associated to $g^{Z}$, then $M_{\widetilde{U}}$ is the mean curvature of $Z$ in the direction of $\widetilde{U}$. Hence (see (4.9), (4.12))

$$
M_{\widetilde{U}}=\sum_{i}\left\langle S\left(e_{i}\right) e_{i}, \widetilde{U}\right\rangle=\frac{1}{2} \sum_{i}\left\langle A_{\widetilde{U}}\left(e_{i}\right), e_{i}\right\rangle
$$

where $\left\{e_{i}\right\}$ is an orthonormal basis for $T Z_{m}$.

(b) Odd dimensional base spaces. Let $Z^{2 l} \rightarrow M^{2(k+l)-1} \stackrel{\pi}{\rightarrow} B^{2 k-1}$ be as in part (a). We assume that $B$ is oriented and spin with Hermitian bundle of spinors $F^{B}$. The Levi Civita connection $\nabla^{B}$ on $T B$ lifts to a connection $\nabla^{F^{B}}$ on $F^{B}$. We also assume that the bundle $T Z \subset T M$ is oriented and spin. Since $T Z$ is endowed with a metric, $g^{Z}$, we can define the Hermitian bundle of spinors, $F^{Z}=F_{+}^{Z} \oplus F_{-}^{Z}$. Then $F^{Z}$ inherits a connection $\nabla^{F^{Z}}$ from the connection $P^{Z} \nabla^{L}$. We can take

$$
F^{M}=F_{+}^{M} \oplus F_{-}^{M}=\pi^{*}\left(F^{B}\right) \otimes F^{Z}=\pi^{*}\left(F^{B}\right) \otimes F_{+}^{z} \oplus \pi^{*}\left(F^{B}\right) \otimes F_{-}^{Z}
$$

which we endow with the tensor product connection $\pi^{*}\left(\nabla^{F^{B}}\right) \otimes \nabla^{F^{Z}}=\nabla^{F^{\prime \prime}}$.

We regard $F^{M}$ as a Clifford module over $T M$ as in $\S 1$. If $X \in \pi^{*}(T B)$ then $X$ acts on $\pi^{*}\left(F^{B}\right) \otimes F^{z}$ as $X \otimes \tau^{F^{Z}}$. If $Y \in T Z$ then $Y$ acts by $1 \otimes Y$. In 
particular, if $f_{1}, \ldots, f_{2 k-1}$ is an orthonormal basis for $\pi^{*}(T B)$ at $m \in M$, then $f_{1} \cdots f_{2 k-1}$ acts like $(-i)^{k} \tau^{F^{\prime}}$. Similarly, if $e_{1}, \ldots, e_{2 l}$ is an orthonormal basis of $T Z$ then $e_{1} \cdots e_{2 l}$ acts as $(-i)^{l} \tau^{F^{Z}}$ so that $f_{1} \cdots f_{2 k-1}, e_{1} \cdots e_{2 l}$ acts on $F^{M}=\pi^{*}\left(F^{B}\right) \otimes F^{Z}$ as $(-i)^{k+l}$.

Let $\xi$ be a Hermitian vector bundle on $M$ with unitary connection, $\nabla^{\xi}$, and curvature $L^{\xi}$.

Let $D_{\varepsilon}\left(=D_{\varepsilon}^{M}\right)$ be the Dirac operator for $g_{\varepsilon}^{M}$ acting on smooth sections of $F^{M} \otimes \xi$. We now give a simple expression for $D_{\varepsilon}$ which was derived in (3.11) of [B1]. Let $\nabla^{\prime \prime}$ denote the connection on $F^{M} \otimes \xi$ defined by

$$
\nabla^{u}=\nabla^{F^{B}}-\frac{1}{2} \sum\left\langle S\left(e_{i}\right) e_{i}, \cdot\right\rangle
$$

where $\left\{e_{i}\right\}$ is a local orthonormal basis for $T Z$; see (4.21), (4.22). Let

$$
T(U, V)=-S(U, V)+S(V, U)
$$

denote the torsion tensor of $\nabla$ (see (4.11). Let $c(T) \in \Lambda^{2}\left(T^{*} B\right) \otimes \operatorname{End}(T Z$ ) be the 2-form which assigns to $U, V$ the odd endomorphism of $T Z$ determined by letting $T(U, V)$ act on $T Z$ by Clifford multiplication. In the formula that follows, $\left\{f_{\alpha}\right\}$ is a local orthonormal basis for $T B$ and we also write $f_{\alpha}$ for the horizontal lift $\tilde{f}_{\alpha}$. The Dirac operator, $D^{Z}$, along the fibers, extends naturally to an operator, $1 \otimes D^{Z}$ on $F^{M}$, which we continue to denote by $D^{Z}$. Then we have

$$
D_{\varepsilon}=\varepsilon^{1 / 2} \sum_{\alpha} f_{\alpha} \nabla_{f_{n}}^{u}+D^{Z}-\frac{\varepsilon}{4} \sum_{\alpha<\beta} f_{\alpha} f_{\beta} c(T)\left(f_{\alpha}, f_{\beta}\right) .
$$

To obtain (4.26) start with the expression for the Dirac operator, $D$, on an arbitrary spin manifold. If $\left\{w_{i}\right\}$ is a local orthonormal frame field, $\left\{s_{j}\right\}$ is the associated local basis of the spin bundle, and $f$ is a smooth function, then $D$ is given locally by

$$
D\left(f s_{j}\right)=\sum_{i} w_{i}(f) w_{i} s_{j}+f \frac{1}{4} \sum_{i k l}\left\langle\nabla_{w_{i}}^{L} w_{l}, w_{k}\right\rangle w_{i} w_{l} w_{k} s_{j} .
$$

If we apply (4.27) to $\left(M, g_{\varepsilon}^{M}\right)$ and use the results of part (a) of this section, then (4.26) follows by a straightforward computation.

Remark 4.28. Of course (4.26) holds for any riemannian submersion of spin manifolds (i.e., the dimensions of the base and fibers can be arbitrary).

We now recall the definition of the Levi Civita superconnection given in [B1, §3]. Let $H_{\infty}=H_{\infty,+} \oplus H_{\infty,-}$ be the bundle $F^{Z} \otimes \xi$ (with infinite dimensional fiber, obtained by the construction discussed at the end of part (a). Let $\widetilde{\nabla}^{u}$ be the unitary connection on $H_{\infty}$ of (4.17).

Definition 4.29. The Levi Civita superconnection, $A_{u}$, on $H_{\infty}$, is the superconnection

$$
A_{u}=\widetilde{\nabla}^{u}+u^{1 / 2} D^{Z}-c(T) / 4 u^{1 / 2} .
$$


If we wish to regard the operator $D^{M}=D$ in (4.26) as acting on sections of $F^{B} \otimes H^{\infty}$, we simply write $\tilde{\nabla}^{u}$ for $\nabla^{u}$. Then we obtain an operator with just the same structure as the operator $D^{\xi}+\underline{V}$ considered in Appendix 1. The operators $D^{Z}$ and $c(T)$ correspond to $V_{0}, V_{2}$ respectively.

It is only a matter of viewpoint whether we write $\nabla^{u}$ or $\tilde{\nabla}^{u}$ in (4.26). However, in view of (4.18), the distinction is important in (4.30).

The local index theorem for families of [B1, Theorems 4.12 and 4.16] implies

$$
\lim _{u \rightarrow 0} \operatorname{tr}_{s}\left(e^{-A_{u}^{2}}\right)=\frac{1}{(2 \pi i)^{l}} \int_{Z} \widehat{A}\left(i R^{Z}\right) \operatorname{tr}\left(e^{-L^{\xi}}\right) .
$$

Also, by Theorem 2.11 of [BGS], we know that as $u \rightarrow 0$

$$
\operatorname{tr}_{s}\left(\left(D^{Z}+c(T) / 4 u\right) e^{-A_{u}^{2}}\right)=0\left(u^{1 / 2}\right)
$$

uniformly on the compact manifold $M$.

From now on, we will assume that $D^{Z}$ is invertible for all fibers, $Z$. Using Duhamel's formula, we find easily that as $u \rightarrow \infty$, the differential form $\operatorname{tr}_{s}\left(\left(D^{Z}+c(T) / 4 u\right) e^{-A_{u}^{2}}\right)$ decays exponentially. Thus, we can make the following definition.

Definition 4.33. $\hat{\eta}$ denotes the form of odd degree on $B^{2 k-1}$

$$
\hat{\eta}=\int_{0}^{\infty} \operatorname{tr}_{s}\left(\left(D^{Z}+c(T) / 4 u\right) e^{-A_{u}^{2}}\right) d u / 2 u^{1 / 2} .
$$

Note that (4.34) has the same structure as the analytic continuation to $s=0$ of (A.1.5) (with $x=u^{1 / 2}$ ). But by (4.32), no analytic continuation is necessary in the geometric case.

We now prove an infinite dimensional analogue of Theorem 2.28 (see also (A.1.5)-(A.1.7)), which generalizes the results of [BF, Theorem 3.16] and [C3, Theorem 4.27].

Theorem 4.35. As $\varepsilon \rightarrow 0, \bar{\eta}\left(D_{\varepsilon}\right)$ has a limit in $R$, which is given by

$$
\lim _{\varepsilon \rightarrow 0} \bar{\eta}\left(D_{\varepsilon}\right)=\frac{1}{(2 \pi i)^{k}} \int_{B} \hat{A}\left(i R^{B}\right) \hat{\eta}
$$

Moreover,

$$
d \hat{\eta}=\frac{1}{(2 \pi i)^{l}} \int_{Z} \hat{A}\left(i R^{Z}\right) \operatorname{tr}\left(e^{-L^{\xi}}\right) .
$$

Proof. If we represent the exponentials by smooth kernels, then by arguing as in the finite dimensional case considered in [Q], we find

$$
\begin{aligned}
\frac{\partial}{\partial u} \operatorname{tr}_{s}\left(e^{-A_{u}^{2}}\right) & =d\left(\operatorname{tr}_{s}\left(\frac{\partial A_{u}}{\partial u} e^{-A_{u}^{2}}\right)\right) \\
& =d\left(\operatorname{tr}_{s}\left(\left(D^{z}+\frac{c(T)}{4 u}\right) e^{-A_{u}^{2}}\right) \frac{1}{2 u^{1 / 2}}\right.
\end{aligned}
$$


(compare the manipulations in [B1, §2]). By using (4.31) and (4.38) we obtain (4.37).

We now concentrate on proving (4.36). We know by [BF, Theorem 2.4] (see also (3.18) above) that

$$
\bar{\eta}\left(D_{\varepsilon}\right)=\frac{1}{2}\left[\operatorname{dim} \operatorname{ker} D_{\varepsilon}+\frac{1}{\sqrt{\pi}} \int_{0}^{\infty} \operatorname{tr}\left(D_{\varepsilon} e^{-D_{\varepsilon}^{2} u}\right) \frac{d u}{2 u^{1 / 2}}\right]
$$

where the integral is convergent at 0 . As in [BF] and [C3], where the case $\operatorname{dim} B=1$ was considered, the argument will consist of three steps.

(i) First we prove that for $\varepsilon$ small enough there is a uniform positive lower bound for the smallest eigenvalue of $D_{\varepsilon}^{2}$. In particular, for such $\varepsilon, \operatorname{ker} D_{\varepsilon}=0$.

(ii) Then we prove that for $u \in(0, T]$, we have uniform convergence,

$$
\begin{aligned}
\lim _{\varepsilon \rightarrow 0} \operatorname{tr}\left(D_{\varepsilon} e^{-D_{\varepsilon}^{2} u}\right)= & \frac{\sqrt{\pi}}{(2 \pi i)^{k}} \int_{B} \widehat{A}\left(i R^{B}\right) \operatorname{tr}_{s}\left(\left(D^{Z}+\frac{c(T)}{4 u}\right) e^{-A_{u}^{2}}\right) \frac{d u}{2 u^{1 / 2}} \\
& +O\left(\varepsilon^{1 / 2}\left(1+T^{N}\right)\right),
\end{aligned}
$$

for some $N$ (which might depend on $k$ ).

(iii) Finally, we use the same argument as in [C3] (see also the beginning of $\S 3$ above) to show that the large time contribution to the integral (4.39) is negligible.

Step (i).

Proposition 4.41. There exists $\lambda_{0}>0$ such that for $\varepsilon$ sufficiently small, the spectrum of $D_{\varepsilon}^{2}$ is bounded below by $\lambda_{0}$. In particular, for $\varepsilon$ small enough, $\operatorname{dim} \operatorname{ker} D_{\varepsilon}=0$.

Proof. Let $p \in B$ and let \|\|$_{1, y}$ be the norm in the Sobolev space, $H^{1}$, of sections of $F^{Z} \otimes \xi$ over $Z=Z_{p}=\pi^{-1}(p)$ which are in $L_{2}$, together with their first derivatives. Since $D^{Z}$ is elliptic and invertible and since $B$ is compact, there exists a constant $C>0$ such that for any $p \in B, s \in \Gamma\left(F^{Z} \otimes \xi\right)$.

$$
C \int_{Z_{p}}\left\|D^{Z} s\right\|^{2} d \mathrm{Vol}_{Z_{p}} \geq\|s\|_{p, 1}^{2}
$$

Of course, this inequality immediately extends to sections of $F^{M} \otimes \xi=\pi^{*}\left(F^{B}\right) \otimes$ $F^{Z} \otimes \xi$. Set

$$
E_{\varepsilon}=\sum_{\alpha} f_{\alpha}\left(\nabla_{f^{\prime \prime}}^{u}-\left(\varepsilon^{1 / 2} / 8\right) f_{\beta} T\left(f_{\alpha}, f_{\beta}\right)\right)
$$

By (4.26)

$$
D_{\varepsilon}=\varepsilon^{1 / 2} E_{\varepsilon}+D^{Z}
$$

and so,

$$
D_{\varepsilon}^{2}=\varepsilon E_{\varepsilon}^{2}+\varepsilon^{1 / 2}\left[E_{\varepsilon}, D^{Z}\right]+\left(D^{Z}\right)^{2}
$$


(where the bracket is in the graded sense). The operator $\left[E_{\varepsilon}, D\right]$ is easily seen to be a first order differential operator which acts fiberwise. Thus, there is a constant $C^{\prime}$ such that

$$
\left|\varepsilon^{1 / 2} \int_{Z_{p}}\left\langle\left[E_{\varepsilon}, D\right] s, s\right\rangle\right| \leq \varepsilon^{1 / 2} C^{\prime}\|S\|_{1, Z_{p}}^{2} \leq \varepsilon^{1 / 2} C^{\prime} C\left\|D^{Z} s\right\|^{2} \text {. }
$$

Since $E_{\varepsilon}$ is selfadjoint, $\varepsilon E_{\varepsilon}^{2}$ is a positive operator. Thus, if $\varepsilon^{1 / 2} C^{\prime} C \leq \frac{1}{2}$,

$$
\begin{aligned}
D_{\varepsilon}^{2} & \geq \varepsilon^{1 / 2}\left[E_{\varepsilon}, D^{Z}\right]+\left(D^{Z}\right)^{2} \\
& \geq\left(1-\varepsilon^{1 / 2} C^{\prime} C\right)\left(D^{z}\right)^{2} \geq \frac{1}{2}\left(D^{Z}\right)^{2} .
\end{aligned}
$$

The proposition follows.

Step (ii). In order to prove the fixed time convergence result, (4.40), we will introduce an auxiliary Grassmann variable $z$ (i.e. $z^{2}=0$ ) which is odd. Thus $z$ anticommutes with $f_{\alpha}, d y_{\alpha}, e_{i}$.

In [BF] (Proposition 2.1) such a variable was introduced in order to give a new proof of the fact that the $\eta$-function of a Dirac operator is regular at $s=0$. It was also used there to obtain the main theorem of this section in case $\operatorname{dim} B=1$.

As previously explained, the reason for introducing the variable $z$ stems from the fact that the expression $u^{1 / 2} D_{\varepsilon} e^{-D_{\varepsilon}^{2} u}$, which controls (4.39), is singular with respect to Getzler's transformation. Once $z$ has been introduced, we can consider instead the part of $\operatorname{tr}\left(e^{-D_{\varepsilon}^{2} u+z D_{\varepsilon} u^{1 / 2}}\right)$ which involves $z$. One way of desingularizing this expression was explained in $\S 3$. It depended on showing that the operator of passing to the auxiliary adiabatic limit could be interchanged with that of passing to the original one.

In this section we will employ an alternate strategy. First we will prove a Lichnerowicz formula (easily derived from the one proved in [BF, Proposition 2.1]) for the operator $D_{\varepsilon}^{2} u-z D_{\varepsilon} u^{1 / 2}$. Although the resulting expression contains a term which behaves singularly with respect to Getzler's transformation, we show that this term can be cancelled by means of an exponential transform which involves the variable $z$ in an essential way.

First we introduce some notation. Let $Q$ be a section of the bundle of linear maps from $T M$ to $\operatorname{End}\left(F^{Z} \otimes \xi\right)$. We use the abbreviation

$$
\begin{aligned}
& \left(\nabla_{e_{i}}+Q\left(e_{i}\right)\right)^{2}=\sum\left(\nabla_{e_{i}}+Q\left(e_{i}\right)\right)^{2}-\nabla_{\sum_{i} \nabla_{c_{i}} e_{i}}-Q\left(\sum_{i} \nabla_{e_{i}} e_{i}\right) \\
& \left(\nabla_{f_{a}}+Q\left(f_{\alpha}\right)\right)^{2}=\sum_{\alpha}\left(\nabla_{f_{a}}+Q\left(f_{\alpha}\right)\right)^{2}-\nabla_{\sum_{n} \nabla_{f_{n}} f_{l l}}-Q\left(\sum_{\alpha} \nabla_{f_{n}} f_{\alpha}\right) .
\end{aligned}
$$

Let $k_{\varepsilon}^{M}$ be the scalar curvature of $M$ for the metric $g_{\varepsilon}^{M}$. In the formulas which follow, we sum over all repeated indices. 
Proposition 4.50. For $u>0, \varepsilon>0$, the following formula holds.

$$
\begin{aligned}
D_{\varepsilon}^{2} u-z D_{\varepsilon} u^{1 / 2}= & -u\left[\nabla_{e_{i}}+\frac{1}{2}\left\langle S\left(e_{i}\right) e_{j}, f_{\alpha}\right\rangle e_{j} \varepsilon^{1 / 2} f_{\alpha}\right. \\
& \left.+\frac{1}{4}\left\langle S\left(e_{i}\right) f_{\alpha}, f_{\beta}\right\rangle \varepsilon^{1 / 2} f_{\alpha} \varepsilon^{1 / 2} f_{\beta}+z e_{i} / 2 u^{1 / 2}\right]^{2} \\
& -u\left[\varepsilon^{1 / 2} \nabla_{f_{\alpha}}+\frac{1}{2}\left\langle S\left(f_{\alpha}\right) e_{i}, f_{\beta}\right\rangle e_{i} \varepsilon f_{\beta}+z f_{\alpha} / 2 u^{1 / 2}\right]^{2} \\
& +\frac{u}{2} e_{i} e_{j} \otimes L^{\xi}\left(e_{i}, e_{j}\right)+u \varepsilon^{1 / 2} f_{\alpha} e_{i} \otimes L^{\xi}\left(f_{\alpha}, e_{i}\right) \\
& +\frac{u}{2} \varepsilon^{1 / 2} f_{\alpha} \varepsilon^{1 / 2} f_{\beta} \otimes L^{\xi}\left(f_{\alpha}, f_{\beta}\right)+\frac{u}{4} k_{\varepsilon}^{M} \\
& +\frac{1}{4} \varepsilon u\left\|\sum S\left(e_{i}\right) e_{i}\right\|^{2}-\varepsilon \frac{u}{2}\left\langle\nabla_{f_{i}} S\left(e_{j}\right) e_{j}, f_{\alpha}\right\rangle .
\end{aligned}
$$

Proof. Let $w_{1} \cdots w_{2(k+l)-1}$ be a locally defined smooth orthonormal frame field on an arbitrary spin manifold and let $\nabla^{L}$ denote the Levi Civita connection. Let $\xi$ be as above. By [BF, Proposition 2.1], we know that

$$
D^{2} u-z D u^{1 / 2}=u\left(\nabla_{w_{i}}^{L}+\frac{1}{2 u^{1 / 2}} z w_{i}\right)^{2}+u \frac{k^{M}}{4}+\frac{u}{2} w_{i} w_{j} \otimes L^{\xi}\left(w_{i}, w_{j}\right)
$$

We apply this formula to $\left(M, g_{\varepsilon}^{M}\right)$ and make the substitution $\nabla^{L}=\nabla+S$. By computing as in $[\mathrm{B} 1,(3.14)]$, we find that

$$
\text { (4.53) } \begin{aligned}
D_{\varepsilon}^{2} u-z D_{\varepsilon} u^{1 / 2}= & -u\left[\nabla_{e_{i}}+\frac{1}{2}\left\langle S\left(e_{i}\right) e_{j}, f_{\alpha}\right\rangle e_{j} \varepsilon^{1 / 2} f_{\alpha}\right. \\
& \left.+\frac{1}{4}\left\langle S\left(e_{i}\right) f_{\alpha}, f_{\beta}\right\rangle \varepsilon^{1 / 2} f_{\alpha} \varepsilon^{1 / 2} f_{\beta}+z e_{i} / 2 u^{1 / 2}\right]^{2} \\
& -u\left[\varepsilon^{1 / 2} \nabla_{f_{n}}+\frac{1}{2}\left\langle S\left(f_{\alpha}\right) e_{i}, f_{\beta}\right\rangle e_{i} \varepsilon f_{\beta}+z f_{\alpha} / 2 u^{1 / 2}\right]^{2} \\
& +\frac{1}{2} u\left\langle S\left(S\left(e_{i}\right) e_{i}\right) e_{j}, f_{\alpha}\right\rangle e_{j} \varepsilon^{3 / 2} f_{\alpha} \\
& +z\left(u^{1 / 2} / 2\right) \varepsilon^{1 / 2} S\left(e_{i}\right) e_{i}+\nabla_{\varepsilon S\left(e_{i}\right) e_{i}} \\
& +\frac{u}{2} e_{i} e_{j} \otimes L^{\xi}\left(e_{i}, e_{j}\right)+\frac{u}{2} \varepsilon^{1 / 2} f_{\alpha} e_{i} \otimes L^{\xi}\left(f_{\alpha}, e_{i}\right) \\
& +\frac{u}{2} \varepsilon^{1 / 2} f_{\alpha} \varepsilon^{1 / 2} f_{\beta} \otimes L^{\xi}\left(f_{\alpha}, f_{\beta}\right)+\frac{u}{4} k_{\varepsilon}^{M} .
\end{aligned}
$$

Note that with respect to [B1, (3.14)], we have cancelled the terms $S\left(f_{\alpha}\right) f_{\alpha}$, since these terms vanish (see (4.12), (4.13) above and the sentence which follows). Also, since $S\left(e_{i}\right) e_{i}$ is horizontal, $\left\langle S\left(S\left(e_{i}\right) e_{i}\right) f_{\alpha}, f_{\beta}\right\rangle=0$.

One easily checks that (4.52) is equivalent to (4.53).

We now turn to the proof of (4.40). First of all, the estimates of [C3, §3] and a straightforward generalization of arguments of $[C 3, \S 4]$ show in studying the left-hand side of (4.39) that we can replace the base space by $R^{2 k-1}$ with a metric which is flat outside a compact set. Similarly, we can assume that the bundle is isometrically a product on that region.

Let $z$ be an odd Grassman variable as described after the proof of Proposition 4.41. If $A$ and $B$ are trace class in End $H_{\infty}$, set

$$
\operatorname{tr}^{2}(A+z B)=z \operatorname{tr}(B) .
$$


As in [BF, §2] (see also (3.12) above), we find that

$$
e^{-D_{\varepsilon}^{2} u+z D_{r} u^{1 / 2}}=e^{-D_{\varepsilon}^{2} u}+z u^{1 / 2} D_{\varepsilon} e^{-D_{\varepsilon}^{2} u}
$$

Thus,

$$
\operatorname{tr}^{z}\left(e^{-D_{\varepsilon}^{2} u+z D_{\varepsilon} u^{1 / 2}}\right)=z u^{1 / 2} \operatorname{tr}\left(D_{\varepsilon} e^{-D_{\varepsilon}^{2} u}\right)
$$

Take $x_{0} \in M$. For $s>0$ let $P_{s}^{\varepsilon, u}\left(x_{0}, \cdot\right)$ be the $C^{\infty}$ kernel, with respect to the volume element of $g_{1}^{M}$, of the operator $e^{s\left(-D_{\varepsilon}^{2} u+z D_{\varepsilon} u^{1 / 2}\right)}$. If $h$ is a smooth section of $F^{M} \otimes \xi$, then

$$
e^{s\left(-D_{\varepsilon}^{2} u+z D_{\varepsilon} u^{1 / 2}\right)}(h)\left(x_{0}\right)=\int_{M} P_{s}^{\varepsilon, u}\left(x_{0}, x\right) h(x) d \operatorname{vol}_{x} .
$$

Let $y_{0}=\pi\left(x_{0}\right)$ and take a system of geodesic coordinates $\left\{y_{\alpha}\right\}$ centered at $y_{0}$. We can assume $\left\{y_{a}\right\}$ is globally defined on $R^{2 k-1}$. By using parallel transport along the horizontal lifts of geodesics in the base we can trivialize the fibration $Z \rightarrow M \rightarrow R^{2 k-1}$. Similarly, we can use parallel transport along such geodesics to trivialize $F^{M} \otimes \xi$.

In what follows, we put $\bar{y}=\sum_{\alpha} y_{\alpha} f_{\alpha}$. As in [BF, Theorem 3.12], we will conjugate the operator $D_{\varepsilon}^{2} u-z D_{z} u^{1 / 2}$ by

$$
e^{z \bar{y} / 2(\varepsilon u)^{1 / 2}}=1+\sum_{\alpha} \frac{z y_{\alpha} f_{\alpha}}{2(\varepsilon u)^{1 / 2}} .
$$

This will introduce a term which cancels the effect of the term $z f_{\alpha} / 2 u^{1 / 2}$ in (4.51). The latter blows up if we apply $G_{(\varepsilon u)^{1 / 2}}$ and let $\varepsilon \rightarrow 0$.

For $x \in M, \pi(x)=y$, set

$$
\widehat{P}_{s}^{\varepsilon, u}\left(x_{0}, x\right)=P_{s}^{\varepsilon, u}\left(x_{0}, x\right) e^{-z \bar{y} / 2(\varepsilon u)^{1 / 2}} .
$$

Clearly,

$$
\begin{aligned}
\frac{\partial}{\partial s} \widehat{P}_{s}^{\varepsilon, u}\left(x_{1}, \cdot\right)= & P_{s}^{\varepsilon, u}\left(-D_{\varepsilon}^{2} u+z D_{\varepsilon} u^{1 / 2}\right) e^{-z \bar{y} / 2(\varepsilon u)^{1 / 2}} \\
= & \widehat{P}_{s}^{\varepsilon, u} e^{z \bar{y} / 2(\varepsilon u)^{1 / 2}}\left(-D_{\varepsilon}^{2} u+z D_{\varepsilon} u^{1 / 2}\right) e^{-z \bar{y} / 2(\varepsilon u)^{1 / 2}}, \\
& \lim _{s \rightarrow 0} P_{s}^{\varepsilon, u}\left(x_{0}, x\right)=\delta_{x_{0}} \otimes I_{F^{\prime \prime} \otimes \xi}
\end{aligned}
$$

(where $I_{F^{M} \otimes \xi}$ denotes the identity transformation).

Since $\pi\left(x_{0}\right)=0$, we have $\widehat{P}_{s}^{\varepsilon, u}\left(x_{0}, x_{0}\right)=P_{s}^{\varepsilon, u}\left(x_{0}, x_{0}\right)$. Thus,

$$
\begin{aligned}
& \operatorname{tr}\left(\widehat{P}_{s}^{\varepsilon, u}\left(x_{0}, x_{0}\right)=\operatorname{tr}\left(P_{s}^{\varepsilon, u}\left(x_{0}, x_{0}\right)\right),\right. \\
& \operatorname{tr}^{z}\left(\widehat{P}_{s}^{\varepsilon, u}\left(x_{0}, x_{0}\right)\right)=\operatorname{tr}^{z}\left(P_{s}^{\varepsilon, u}\left(x_{0}, x_{0}\right)\right) .
\end{aligned}
$$


By using (4.51) (of Proposition 4.50) it follows easily that

$$
\begin{aligned}
& e^{z \bar{y} / 2(\varepsilon u)^{1 / 2}}\left(D_{\varepsilon}^{2} u-z D_{\varepsilon} u^{1 / 2}\right) e^{-z \bar{y} / 2(\varepsilon u)^{1 / 2}} \\
& =-u\left[\nabla_{e_{i}}+\frac{1}{2}\left\langle S\left(e_{i}\right) e_{j}, f_{\alpha}\right\rangle e_{j} \varepsilon^{1 / 2} f_{\alpha}+\frac{1}{4}\left\langle S\left(e_{i}\right) f_{\alpha}, f_{\beta}\right\rangle \varepsilon^{1 / 2} f_{\alpha} \varepsilon^{1 / 2} f_{\beta}\right. \\
& \left.+\frac{1}{2 u^{1 / 2}} z e_{i}+\frac{1}{2 u^{1 / 2}}\left\langle S\left(e_{i}\right) e_{j}, \bar{y}\right\rangle z e_{j}-\frac{1}{2 u^{1 / 2}}\left\langle S\left(e_{i}\right) \bar{y}, f_{\beta}\right\rangle z \varepsilon^{1 / 2} f_{\beta}\right]^{2} \\
& -u\left[\varepsilon^{1 / 2} \nabla_{f_{i n}}+\frac{1}{2}\left\langle S\left(f_{\alpha}\right) e_{i}, f_{\beta}\right\rangle e_{i} \varepsilon f_{\beta}\right. \\
& +\frac{1}{2 u^{1 / 2}} z f_{\alpha}-\frac{1}{2 u^{1 / 2}} f_{\alpha}\left(y^{\beta}\right) z f_{\beta}+\frac{1}{2 u^{1 / 2}}\left\langle\nabla_{f_{u}} f_{\gamma}, \bar{y}\right\rangle z f_{\gamma} \\
& \left.+\frac{\varepsilon^{1 / 2}}{2 u^{1 / 2}}\left\langle S\left(f_{\alpha}\right) e_{i}, \bar{y}\right\rangle z e_{i}\right]^{2} \\
& +\frac{u}{2} e_{i} e_{j} \otimes L^{\xi}\left(e_{i}, e_{j}\right)+u \varepsilon^{1 / 2} f_{\alpha} e_{i} \otimes L^{\xi}\left(f_{\alpha}, e_{i}\right) \\
& +\frac{u}{2} \varepsilon^{1 / 2} f_{\alpha} \varepsilon^{1 / 2} f_{\beta} \otimes L^{\xi}\left(f_{\alpha}, f_{\beta}\right)+\frac{u}{4} k_{\varepsilon}^{M} \\
& +\frac{\varepsilon u}{4}\left\|\sum S\left(e_{i}\right) e_{i}\right\|^{2}-\frac{\varepsilon u}{2}\left\langle\nabla_{f_{n}} S\left(e_{j}\right) e_{j}, f_{\alpha}\right\rangle \\
& -u^{1 / 2} z e_{i} \otimes L^{\xi}\left(\bar{y}, e_{i}\right)-u^{1 / 2} \varepsilon^{1 / 2} z f_{\alpha} \otimes L^{\xi}\left(\bar{y}, f_{\alpha}\right) \text {. }
\end{aligned}
$$

Note that with the exception of the terms

$$
-\frac{1}{2 u^{1 / 2}} f_{c r}\left(y_{\beta}\right) z f_{\beta}+\frac{1}{2 u^{1 / 2}}\left\langle\nabla_{f_{u}} f_{y}, \bar{y}\right\rangle z f_{\gamma},
$$

the terms which appear in (4.64) but not in (4.51) arise as consequences of the relation

$$
e^{z \bar{y} / 2(\varepsilon u)^{1 / 2}} f_{\alpha} e^{-z \bar{y} / 2(\varepsilon u)^{1 / 2}}=f_{\alpha}-z y_{\alpha} /(\varepsilon u)^{1 / 2} .
$$

The terms in (4.65) arise from conjugating $\nabla_{f_{\text {. }}}$.

Now apply Getzler's transformation $G_{(\varepsilon u)^{1 / 2}}$ to the expression on the righthand side of (4.64) and let $\varepsilon \rightarrow 0$. Since $\bar{y}$ gets replaced by $(\varepsilon u)^{1 / 2} \bar{y}$, we see that all the newly created terms in (4.64) drop out with the exception of those in (4.65). Since $\left\langle\nabla_{f_{\beta}} f_{\alpha}, f_{\gamma}\right\rangle=0(y)$, we have

$$
G_{(\varepsilon u)^{1 / 2}}\left(\frac{1}{2 u^{1 / 2}}\left\langle\nabla_{f_{u}} f_{y}, \bar{y}\right\rangle z f_{y}\right)=0\left(\varepsilon^{1 / 2} u^{1 / 2}\right),
$$

and the second term in (4.65) also drops out. Most importantly, since $f_{\alpha}\left(y_{\beta}\right)=$ $\delta_{\alpha \beta}+0\left(y^{2}\right)$, the first term in (4.65) cancels the singular term $z f_{\alpha} / 2 u^{1 / 2}$, which came from (4.51) and which appears in the second square bracket in (4.64). All 
remaining terms in (4.64) approach finite limits and we find that

$$
\begin{aligned}
\lim _{\varepsilon \rightarrow 0} G_{(\varepsilon u)^{1 / 2}}\left[e^{z y / 2(\varepsilon u)^{1 / 2}}\left(u D_{\varepsilon}^{2}-z u^{1 / 2} D_{\varepsilon}\right) e^{-z y / 2(\varepsilon u)^{1 / 2}}\right] & \\
=-u & {\left[\nabla_{e_{i}}+\frac{1}{2 u^{1 / 2}}\left\langle S\left(e_{i}\right) e_{j}, f_{\alpha}\right\rangle e_{j} d y_{\alpha}\right.} \\
& \left.\quad+\frac{1}{4 u}\left\langle S\left(e_{i}\right) f_{\alpha}, f_{\beta}\right\rangle d y_{\alpha} d y_{\beta}+z e_{i} / 2 u^{1 / 2}\right]^{2} \\
& -\left(\partial_{\alpha}+\frac{1}{8}\left\langle R^{B}\left(f_{\alpha}, f_{\beta}\right) f_{\gamma}, f_{\delta}\right\rangle y_{\beta} d y_{\gamma} d y_{\delta}\right)^{2} \\
+ & \frac{u}{2} e_{i} e_{j} \otimes L^{\xi}\left(e_{i}, e_{j}\right)+u^{1 / 2} d y_{\alpha} e_{i} \otimes L^{\xi}\left(f_{\alpha}, e_{i}\right) \\
+ & \frac{1}{2} d y_{\alpha} d y_{\beta} \otimes L^{\xi}\left(f_{\alpha}, f_{\beta}\right)+\frac{u}{4} k^{Z}
\end{aligned}
$$

We have

$$
\mathscr{H}=-\left(\partial_{\alpha}+\frac{1}{8}\left\langle R^{B}\left(f_{\alpha}, f_{\beta}\right) f_{\gamma}, f_{\delta}\right\rangle y^{\beta} d y^{\gamma} d y^{\delta}\right)^{2}
$$

where $\mathscr{H}$ is the generalized Hermite operator on $B$ which was considered by Getzler and which appeared in $\S 2$. One easily checks that the expression in (4.68) is equal to

$$
\mathscr{H}+A_{u}^{2}-z\left(u^{1 / 2} D^{Z}+c(T) / 4 u^{1 / 2}\right)
$$

(see [BGS, Proposition 2.10] for details). Evaluating $\operatorname{tr}^{z}$ at the origin gives

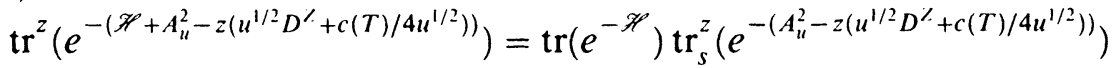

$$
\begin{aligned}
& \operatorname{tr}\left(e^{-\not{H}}\right)=\frac{(-i)^{l}}{\pi^{l-1 / 2} 2^{l}} \widehat{A}\left(i R^{B}\right) .
\end{aligned}
$$

Also, by Duhamel's formula,

$$
\operatorname{tr}_{s}\left(e^{-A_{u}^{2}+z\left(u^{1 / 2} D^{\prime}+c(T) / 4 u^{1 / 2}\right)}\right)=\operatorname{tr}_{s}\left(e^{-A_{u}^{2}}\right)+z \operatorname{tr}_{s}\left[\left(u^{1 / 2} D^{Z}+c(T) / 4 u^{1 / 2}\right) e^{-A_{u}^{2}}\right] .
$$

Now (using (4.55)) the same arguments as in [G] (see also [B1]) show that (4.74)

$$
\frac{1}{2 \sqrt{\pi} u^{1 / 2}} \operatorname{tr}\left(D_{\varepsilon} e^{-D_{r}^{2} u}\right) \rightarrow \frac{1}{(2 \pi i)^{l}} \int_{B} \widehat{A}\left(i R^{B}\right) \operatorname{tr}_{s}\left[\left(D^{Z}+\frac{c(T)}{4 u}\right) e^{-A_{u}^{2}}\right] \frac{1}{2 u^{1 / 2}}
$$

uniformly, for $u \in[\delta, T]$.

To see that the convergence is actually uniform for $u \in[0, T]$, it suffices to show that the coefficient, $c_{\varepsilon}$, in the small time expansion,

$$
\frac{1}{2 \sqrt{\pi} u^{1 / 2}} \operatorname{tr}\left(D_{\varepsilon} e^{-D_{c}^{2} u}\right)=c_{\varepsilon}+0(u)
$$


approaches a limit and that the remainder term remains uniformly bounded by $c \cdot u$, independent of $\varepsilon$. Now

$$
z \frac{1}{2 \sqrt{\pi} u^{1 / 2}} \operatorname{tr}\left(D_{\varepsilon} e^{-D_{\varepsilon}^{2} u}\right)=\frac{1}{2 \sqrt{\pi} u^{3 / 2}} \operatorname{tr}^{z}\left(e^{-u\left(D_{\varepsilon}^{2}-z D_{\varepsilon}\right)}\right) .
$$

Taking $u=1$ in (4.68) gives

$$
\text { (4.77) } \begin{aligned}
\lim _{\varepsilon \rightarrow 0} G_{\varepsilon^{1 / 2}}\left[e^{z \ddot{y} / 2 \varepsilon^{1 / 2}}\left(D_{\varepsilon}^{2}-z D_{\varepsilon}\right) e^{-z \ddot{y} / 2 \varepsilon^{1 / 2}}\right] & \stackrel{\text { def }}{=} \lim _{\varepsilon \rightarrow 0} \mathscr{L}_{\varepsilon} \\
& =\mathscr{L}_{0} \\
& \stackrel{\text { def }}{=} \mathscr{H}+A_{1}^{2}-z\left(D^{z}+c(T) / 4\right) .
\end{aligned}
$$

Since the asymptotic expansion of $\operatorname{tr}\left(e^{-u \mathscr{L}_{\varepsilon}}\right)$ at the origin depends on the local symbol of $\mathscr{L}_{\varepsilon}$, it follows that the coefficients in this expansion converge as $\varepsilon \rightarrow 0$, to those in the expansion for $\operatorname{tr}\left(e^{-u \mathscr{L}_{0}}\right)$ and that the remainder terms are bounded by $c \cdot u$ independent of $\varepsilon$. By putting this together with (4.75) and (4.76) we see that $c_{\varepsilon} \rightarrow c_{0}$ and that the remainder is bounded by $c \cdot u$ independent of $\varepsilon$.

Remark 4.78. The above argument also shows that the expansion of $\operatorname{tr}\left(e^{-\mathscr{L}_{0} u}\right)$ starts with the term $c_{0}$ and hence does not contain singular terms. By using the fact that only terms which saturate the Grassmann variables $d y^{1} \cdots d y^{2 k-1}$ can contribute to the trace, it is easy to see by degree counting that

$$
\operatorname{tr}^{z}\left(e^{-\left[\not{P}+A_{u}^{2}-z\left(u^{1 / 2} D^{\prime}+c(T) / 4 u^{1 / 2}\right)\right]}\right)=\operatorname{tr}\left(e^{-u\left[\mathscr{P}+A_{1}^{2}-z\left(D^{\prime}+c(T)\right)\right]}\right) / u^{1 / 2} .
$$

Thus,

$$
\widehat{A}\left(i R^{B}\right) \operatorname{tr}\left[\left(D^{Z}+c(T) / 4 u\right) e^{-A_{u}^{2}}\right]=0\left(u^{1 / 2}\right) .
$$

Since, in (4.80), the metric on $B$ is arbitrary, it is not hard to see that this implies (4.32).

Note that in (4.74) we did not include any estimate on the remainder term. However, such an estimate can be obtained from a straightforward generalization of the argument given in [C3], for the case $\operatorname{dim} B=1$. Namely, we treat $M$ as a perturbation of the product, $R^{2 k-1} \times Z^{2 l}$ and iterate Duhamel's principle $(2 k+1)$ times. In this way, for fixed $u \in[1, T]$, we obtain an expansion

$$
\operatorname{tr}\left(D_{\varepsilon} e^{-D_{\varepsilon}^{2} u}\right)=\sum_{i=0}^{2 k-1} a_{i} \varepsilon^{(i-2 k+1) / 2}+0\left(\varepsilon^{1 / 2} T^{N}\right) .
$$

The coefficients $a_{i}=a_{i}(u)$ also satisfy $\left|a_{i}\right| \leq T^{N}$. The derivation of (4.81) (which would be tedious to write out in detail) is completely analogous to the one given in [C3, pp. 194-197]. Note that the estimates of [C3, pp . 192-194] on which that discussion is based, are valid in all dimensions.

If we use (4.74), it follows that the singular terms in (4.81) vanish and that the constant term must coincide with the right-hand side of (4.74). Thus, (4.74) and (4.81) give (4.40). 
Step (iii). As explained at the beginning of the proof, relation (4.40) yields Theorem 4.35 by using the argument of [C3, p. 181] (see also (3.3) above) to show that the large time contribution to the integral in (4.39) vanishes in the limit, $\varepsilon \rightarrow 0$.

Remark 4.82. We point out that $\operatorname{tr}\left(D_{\varepsilon} e^{\left.-D_{\varepsilon}^{2} t\right)}\right.$ is actually uniformly exponentially decreasing (independent of $\varepsilon$ ). In fact the inequality

$$
\lambda^{1 / 2} e^{-\lambda u} \leq c \cdot \frac{e^{-\lambda u / 2}}{u^{1 / 2}},
$$

together with the spectral theorem gives, for say $u \geq 4$,

$$
\begin{aligned}
\operatorname{tr}\left(D_{\varepsilon} e^{-D_{\varepsilon}^{2} u}\right) & \leq c \cdot \operatorname{tr}\left(e^{-D_{\varepsilon}^{2} u / 2}\right) \\
& \leq c \cdot e^{-\lambda_{0} u / 4} \operatorname{tr}\left(e^{-D_{\varepsilon}^{2}}\right),
\end{aligned}
$$

with $\lambda_{0}$ as in Proposition 4.41. Since the metrics, $g_{\varepsilon}$, have uniformly bounded geometry and volumes which grow like $\varepsilon^{-(2 k-1) / 2}$, by using standard estimates on kernels, we get

$$
\operatorname{tr}\left(e^{-D_{c}^{2}}\right) \leq c \varepsilon^{-(2 k-1) / 2}
$$

Thus,

$$
\operatorname{tr}\left(D_{\varepsilon} e^{-D_{\varepsilon}^{2} u}\right) \leq c e^{-\lambda_{0} u / 4} \varepsilon^{-(2 k-1) / 2}
$$

On the other hand, the integrand in (4.34) is bounded by $c^{\prime} e^{-\lambda_{1} u}$, for some $\lambda_{1}>0$. Combining this with the error estimate $0\left(\varepsilon^{1 / 2} T^{N}\right)($ see $(4.81)$ and the discussion which follows) gives for $u \geq 4$,

$$
\operatorname{tr}\left(D_{\varepsilon} e^{-D_{\varepsilon}^{2} u}\right) \leq c\left(e^{-\lambda_{1} u}+\varepsilon^{1 / 2} u^{N}\right)
$$

By using (4.86) for $\varepsilon^{1 / 2} \geq e^{-\lambda_{0} u / 8(2 k-1)}$ and (4.87) for $\varepsilon^{1 / 2} \leq e^{-\lambda_{0} u / 8(2 k-1)}$, we obtain the required exponential decay.

We point out that the uniform exponential decay was also observed in the corresponding contexts of [B2] and [BF].

Remark 4.88. It is also convenient to have a restatement of Theorem 4.35 in which the characteristic forms represent unnormalized characteristic classes. Let $[\hat{\eta}]_{2 j-1}$ denote the component in degree $2 j-1$ of the odd form $\hat{\eta}$. Put

$$
\tilde{\eta}=\sum \frac{1}{(2 \pi i)^{j}}[\hat{\eta}]_{2 j-1} .
$$

Then (4.36) becomes

$$
\lim _{\varepsilon \rightarrow 0} \eta\left(D_{\varepsilon}\right)=\int_{B} \widehat{A}\left(\frac{R^{B}}{2 \pi}\right) \tilde{\eta}
$$


where $\hat{A}\left(R^{B} / 2 \pi\right)$ represents the $\hat{A}$-class of $B$. Moreover,

$$
d \tilde{\eta}=\int_{Z} \widehat{A}\left(R^{Z} / 2 \pi\right) \operatorname{tr}\left(e^{(i / 2 \pi) L^{\xi}}\right)
$$

where $\widehat{A}\left(R^{Z} / 2 \pi\right)$ represents the $\hat{A}$-class of $T Z$ and $\operatorname{tr}\left(e^{(i / 2 \pi) L^{\xi}}\right)$ represents the Chern character of $\xi$.

(c) Even dimensional base spaces. We consider a riemannian submersion, $Z^{2 l-1} \rightarrow B^{2 k}$, of closed oriented spin manifolds as in part (b). But now $\operatorname{dim} B$ is even and $\operatorname{dim} Z$ is odd. We identify the Hermitian bundle of spinors, $F^{M}$, with $\pi^{*}\left(F^{B}\right) \oplus F^{Z}$ and let $f_{\alpha}, e_{i}$, act by $f_{\alpha} \otimes 1, \tau^{F^{B}} \otimes e_{i}$ respectively. We also introduce a Hermitian bundle with unitary connection and curvature $L^{\xi}$. Then we define $D_{\varepsilon}, \bar{\eta}\left(D_{\varepsilon}\right), c(T), A_{u}$ just as in $\S 4(\mathrm{~b})$. Of course, in defining $A_{u}$, we still assume $d y^{\alpha}, e_{i}$ anticommute (which fits with the formalism of [Q, $\left.\S 5\right]$. The same argument as in [BGS, Theorem 2.11], shows that as $u \rightarrow 0$,

$$
\operatorname{tr}^{\text {even }}\left[\left(D^{Z}+c(T) / 4 u\right) e^{-A_{u}}\right]=0\left(u^{1 / 2}\right) .
$$

The adiabatic limit, $\lim _{\varepsilon \rightarrow 0} \bar{\eta}\left(D_{\varepsilon}\right)$, exists in $R / Z$. The argument is the same as that given in part (a).

Now assume that $D^{Z}$ is invertible for all fibers $Z$.

Definition 4.93. $\hat{\eta}$ denotes the form of even degree,

$$
\hat{\eta}=\frac{1}{\sqrt{\pi}} \int_{0}^{\infty} \operatorname{tr}^{\text {even }}\left[\left(D^{Z}+c(T) / 4 u\right) e^{-A_{u}^{2}}\right] d u / 2 u^{1 / 2} .
$$

Then we have the following infinite dimensional analogue of Theorem 2.35.

Theorem 4.95. As $\varepsilon \rightarrow 0, \hat{\eta}\left(D_{\varepsilon}\right)$ has a limit in $R$, which is given by

$$
\lim _{\varepsilon \rightarrow 0} \hat{\eta}\left(D_{\varepsilon}\right)=\frac{1}{(2 \pi i)^{k}} \int_{B^{2 k}} \widehat{A}\left(i R^{B}\right) \hat{\eta} .
$$

Moreover,

$$
d \hat{\eta}=\frac{1}{(2 \pi i)^{l}} \int_{Z^{2 l-1}} \widehat{A}\left(i R^{Z}\right) \operatorname{tr}\left(e^{-L^{\xi}}\right) .
$$

Proof. The proof is formally identical with that of Theorem 4.35. It is left to the reader.

Remark 4.98. The component $[\hat{\eta}]_{0}$ of $\hat{\eta}$, which is of degree 0 , is just the $\eta$ invariant of the fiber. In this case (4.87) expresses $[d \hat{\eta}]_{0}$ in local terms as in [APS].

Remark 4.99. Theorems 4.35 and 4.95 are actually equivalent. Let $S^{1}, S^{1}$ be two copies of the unit circle and let $\gamma$ be the Hermitian line bundle over $S^{1} \times S^{1}$ whose restriction to $\theta \times \underline{S}^{1}$ has holonomy $\theta \quad(0 \leq \theta \leq 2 \pi)$. Then $c_{1}(\gamma)$ is the generator of $H^{2}\left(S^{1} \times \underline{S}^{1}, Z\right)$. Given $Z \rightarrow M \rightarrow B$ and a twisting bundle $\xi$, we pass to $Z \times S^{1} \rightarrow M \times S^{1} \times \underline{S}^{1} \rightarrow B \times \underline{S}^{1}$ and the bundle $\xi \times \gamma$ (compare 
[BF, Theorem 3.16]). In this way, we replace the case in which the base space has dimension $n$ by that in which it has dimension $n+1$ (without changing the $\eta$-invariant).

Remark 4.100. If we put

$$
\tilde{\eta}=\sum \frac{1}{(2 \pi i)^{j}}[\eta]_{2 j},
$$

then (4.96), (4.97) get replaced by

$$
\begin{aligned}
& \lim _{\varepsilon \rightarrow 0} \bar{\eta}\left(D_{\varepsilon}\right)=\int_{B^{2 k}} \widehat{A}\left(R^{B} / 2 \pi\right) \tilde{\eta}, \\
& d \tilde{\eta}=\int_{Z^{2 l-1}} \widehat{A}\left(R^{Z} / 2 \pi\right) \operatorname{tr}\left(e^{(i / 2 \pi) L^{\xi}}\right) .
\end{aligned}
$$

(d) The case in which the Dirac operator on the fiber is not always invertible. Even if $D^{Z}$ is not always invertible, we still know that the adiabatic limit, $\lim _{\varepsilon \rightarrow 0} \eta\left(D_{\varepsilon}\right)$, exists in $R / Z$. In this case our discussion can be modified along the lines of that of $[\mathrm{BF}]$ and [C3], in which 1-dimensional base spaces were considered. Essentially, one deforms to a situation in which the operator on the fiber (suitably modified) is invertible. The general result is somewhat messy to state. However, the case in which $\operatorname{ker} D^{Z}$ is a subbundle is particularly important and will be discussed in detail elsewhere. Note that this case includes that of the signature operator. For this operator the adiabatic limit of the $\eta$ invariant exists in $R$, since there is no spectral flow (see also [D] for further discussion). Here we will consider only the following simplest special case.

Assume that $\operatorname{dim} \operatorname{ker} D^{Z}$ is constant and that the subbundle $\operatorname{ker} D^{Z} \subset H_{\infty}=$ $F^{Z} \otimes \xi$ is parallel with respect to the unitary connection $\nabla^{\prime \prime}$. Assume further that the endomorphism $\frac{1}{4} \sum_{\alpha<\beta} c(T)\left(f_{\alpha}, f_{\beta}\right) d y_{\alpha} d y_{\beta}$ preserves the splitting, $H_{\infty}=\operatorname{ker} D^{Z} \oplus\left(\operatorname{ker} D^{Z}\right)^{\perp}$. Let $\eta\left(D_{\varepsilon}^{\mathcal{J}}\right)$ denote the $\eta$-invariant of $D^{M}$ (for the metric $g_{\varepsilon}^{M}$ ) restricted to the subbundle $\mathscr{I}=\operatorname{ker} D^{Z}$. Then we have the following essentially obvious result.

\section{Theorem 4.104.}

$$
\lim _{\varepsilon \rightarrow 0} \eta\left(D_{\varepsilon}\right)-\eta\left(D_{\varepsilon}^{\mathscr{Y}}\right)=\int_{B^{2 k}} \widehat{A}\left(R^{B} / 2 \pi\right) \tilde{\eta}
$$

Moreover, $\lim _{\varepsilon \rightarrow 0} \eta\left(D_{\varepsilon}\right), \lim _{\varepsilon \rightarrow 0} \eta\left(D_{\varepsilon}^{\mathcal{J}}\right)$ exist individually in $R / Z$.

One important case in which the assumptions given above are verified is the case of flat torus bundles with affine holonomy (which arise in connection with cusps of $Q$-rank 1 locally symmetric spaces). This will be discussed at greater length elsewhere. Another case is that of $S^{1}$ bundles with holonomy which acts by isometries. 


\section{APPENDIX 2. Multifibrations}

We briefly describe a generalization of the results of parts (b)-(d) of $\S 4$ to the case of multifibrations. As explained below in Example A.2.7 this generalization is significant for applications.

We consider a sequence of fibrations of compact riemannian manifolds

$$
\begin{array}{lll}
F_{0} & \rightarrow & B_{0}=M \\
F_{1} & \rightarrow & B_{1} \\
& & \downarrow \pi_{0} \\
& & \vdots \\
& & \downarrow \\
F_{N-1} & \rightarrow & B_{N-1} \\
& & \downarrow \\
& & B_{N}
\end{array}
$$

Thus, any composition $\pi_{j} \circ \cdots \circ \pi_{i}$ is also the projection map of a fibration $B_{i} \stackrel{\pi_{j} 0 \cdots \circ \pi_{i}}{\rightarrow} B_{j+1}$, whose fiber, $\mathscr{F}_{i, j}$ is the total space of a multifibration

$$
\begin{array}{rll}
F_{i} & \rightarrow & \mathscr{F}_{i, j} \\
F_{i+1} & \rightarrow & \mathscr{F}_{i+1, j} \\
& & \downarrow \\
& \\
& & 0 \\
& & \circ \\
& & \downarrow \\
F_{j} & \simeq \mathscr{F}_{j, j}
\end{array}
$$

We assume that the maps, $\pi_{0}, \ldots, \pi_{N-1}$, are riemannian submersions. We also assume that $B_{N}$ has a spin structure and that for each fibration $F_{i} \rightarrow B_{i} \rightarrow$ $B_{i+1}$, the tangent bundle along the fibers has a spin structure. Then there are induced spin structures on $M=B_{0}, \ldots, B_{N-1}$ as well.

Finally, let $\xi$ be a Hermitian vector bundle with unitary connection over $M=B_{0}$.

Let

$$
T M=H_{N} \oplus H_{N-1} \oplus \cdots \oplus H_{1} \oplus T F_{0}
$$

be the orthogonal splitting of $T M$ induced by (A.2.1). Thus, $\pi_{i-1} \circ \cdots \circ \pi_{0}$ maps $H_{i}$ isomorphically onto $T F_{i} \subset T B_{i}$. We now put $(\varepsilon)=\left(\varepsilon_{1}, \ldots, \varepsilon_{N}\right)$ and consider the metric $g_{(\varepsilon)}^{M}$ on $M$, obtained by blowing up the metric on $H_{i}$ by a factor $\left(\varepsilon_{1} \cdots \varepsilon_{i}\right)^{-1}$.

Let $D_{(\varepsilon)}$ denote the Dirac operator of $g_{(\varepsilon)}$ with coefficients in $\xi$. Let $\tilde{\eta}^{F_{0}}$ denote the $\tilde{\eta}$-form for the fibration $F_{0} \rightarrow B_{0} \rightarrow B_{1}$ and let $\widehat{A}\left(R^{F_{i}} / 2 \pi\right)$ denote the $\hat{A}$-form of the tangent bundle along the fibers for the fibration $F_{i} \rightarrow B_{i} \rightarrow$ $B_{i+1}$. Let $\int_{i}$ denote integration over the fiber $F_{i, N-1}$, where $i=1, \ldots, N-1$. 
Theorem A.2.4. Let $M^{n}$ be odd dimensional and assume $D^{F_{0}}$ is invertible for all fibers $F_{0}$. Then

$$
\lim _{(\varepsilon) \rightarrow 0} \bar{\eta}\left(D_{(\varepsilon)}\right)=\int_{B_{N}} \hat{A}\left(\frac{R^{B_{N}}}{2 \pi}\right)\left[\prod_{i=1}^{N-1} \int_{i} \hat{A}\left(\frac{R^{F_{i}}}{2 \pi}\right)\right] \int_{1} \tilde{\eta}^{F_{0}} .
$$

Remark A.2.6. The thrust of Theorem A.2.4 is that the limit exists independent of the manner in which $\varepsilon_{1}, \ldots, \varepsilon_{N}$ tend to zero. The explicit formula in (A.2.5) is obtained by applying the results of $\S 4$ to the case $\lim _{\varepsilon_{s} \rightarrow 0} \cdots \lim _{\varepsilon_{1} \rightarrow 0}$. The orem A.2.4 can be proved along the same lines of the arguments of $\S 3$ and/or $\S 4$.

Example A.2.7. Let $M$ be the cross section of a cusp in a $Q$-rank 1 locally symmetric space of finite volume. Then $M$ is the total space of a bundle with a locally symmetric space as base space and a nilmanifold as fiber. Thus, using the nilpotent structure, $M$ can be viewed as the total space of a multifibration. If we let $M$ move out to infinity and rescale the induced metric in such a way that the size of the smallest fiber, $F_{0}$, stays fixed, we obtain a family of metrics to which Theorem A.2.4 applies (actually one must take into account the fact that $D^{F_{0}}$ is not invertible in these examples; compare $\S 4(\mathrm{~d})$ ).

The special case of torus bundles over tori, $T^{2 k} \rightarrow \sum^{4 k-1} \rightarrow T^{2 k-1}$ has been considered in [A, ADS, M1]. The adiabatic limit of the $\eta$-invariant is shown to be given by the value at zero of a certain (Shimizu) $L$-function. For the case $k=1$, this result was rederived in [A] and in [C3], as a consequence of the main results of $[B F]$ and $[C 3]$.

By starting from the results of the present paper, one can easily show that for $k$ arbitrary, $\lim _{\varepsilon \rightarrow 0}$ of $\eta\left(D_{\varepsilon}, s\right)$ is of the form $L(s) f(s)$ for $s>-2$. Z. D. Liu has verified that $f(0)=1$, which implies $\lim _{\varepsilon \rightarrow 0} \eta\left(D_{\varepsilon}\right)=L(0)$. These considerations also extend to the general $Q$-rank 1 case treated by $W$. Müller, by means of the Selberg trace formula; see [M2]. This case will be discussed further from our viewpoint elsewhere.

The following result is very closely related to Theorem A.2.4. Let $\tilde{\eta}^{\mathscr{F}_{0 . N-1}}$ denote the $\tilde{\eta}$-form for the fibration $B_{0} \rightarrow B_{N}$.

Theorem A.2.8.

$$
\lim _{(\varepsilon) \rightarrow 0} \tilde{\eta}^{\mathscr{F}_{0 . N-1}}=\left[\prod_{i=1}^{N} \int_{i} \hat{A}\left(\frac{R^{F_{i}}}{2 \pi}\right)\right] \int_{1} \tilde{\eta}^{F_{0}} .
$$

Remark A.2.10. Of course, there are also statements corresponding to (A.2.5), (A.2.9) for supertraces of heat kernels. Moreover, these statements actually imply (A.2.5), (A.2.9) via the discussion of $\S 3$.

\section{REFERENCES}

[A] M. F. Atiyah, The logarithm of the Dedekind $\eta$-function, Math. Ann. 278 (1987), 335-380.

[ADS] M. F. Atiyah, H. Donnelly, and I. M. Singer, Eta invariants, signature defects of cusps, and values of L-functions, Ann. of Math. (2) 118 (1983), 131-177. 
[APS] M. F. Atiyah, V. K. Patodi, and I. M. Singer, Spectral asymmetry and Riemannian geometry, I, Math. Proc. Cambridge Philos. Soc. 77 (1975), 43-69.

[AS] M. F. Atiyah and I. M. Singer, Dirac operators coupled to vector potentials, Proc. Nat. Acad. Sci. U.S.A. 81 (1984), 2597.

[BaM] D. Barbasch and $\mathrm{H}$. Moscovici, $L_{2}$-index theorem and the Selbert trace formula, J. Funct. Anal. 53 (1983), 151-201.

[B1] J.-M. Bismut, The Atiyah-Singer Index Theorem for families of Dirac operators: two heat equation proofs, Invent. Math. 83 (1986), 91-151.

[B2] __, Transgressed Chern forms for Dirac operators, J. Funct. Anal. 77 (1988), 32-50.

[BC1] J. M. Bismut and J. Cheeger, Invariants êta et indice des familles pour des varietes a bord, C. R. Acad. Sci. Paris Sér I. 305 (1987), 127-130.

[BC2] __ Families index for manifolds with boundary, superconnections and cones, J. Funct. Anal. (to appear).

[BF] J. M. Bismut and D. S. Freed, The analysis of elliptic families: Dirac operators, eta invariants, and the holonomy theorem of Witten, Comm. Math. Phys. 107 (1986), 103-163.

[BGS] J. M. Bismut, H. Gillet, and C. Soulé, Analytic torsion and holomorphic determinant bundles, II; Direct images and Bott-Chern forms, Comm. Math. Phys. 115 (1988), 79-126.

[C1] J. Cheeger, On the spectral geometry of spaces with cone-like singularities, Proc. Nat. Acad. Sci. USA, Vol. 76, No. 5, pp. 2103-2106, May 1979.

[C2] __ Spectral geometry of singular Riemannian spaces, J. Differential Geom. 18 (1983), 575657.

[C3] _ n-invariants, the adiabatic approximation and conical singularities, J. Differential Geom. 26 (1987), 175-221.

[Chou] A. Chou, The Dirac operator on spaces with conical singularities and positive scalar curvature, Trans. Amer. Math. Soc. 28 (1985), 1-40.

[Co] A. Connes, Noncommutative differential geometry, Inst. Haute Études Sci. Publ. Math. 62 (1986) 41-144.

[CoM] A. Connes and H. Moscovici, Transgression of the Chern character and cyclic cohomology, C. R. Acad. Sci. Paris Sér. I 303 (1986), 913-918.

[D] X. Dai, Ph.D. thesis, SUNY at Stony Brook (in preparation).

[G] E. Getzler, A short proof of the Atiyah-Singer Index Theorem, Topology 25 (1986), 111-117.

[Gil] P. Gilkey, Invariance theory, the heat equation, and the Atiyah-Singer index theorem, Math. Lecture Series., No. 11, Publish or Perish, Boston, 1984.

[M1] W. Müller, Signature defects of cusps of Hilbert modular varieties and values of L-series at $s=1$, J. Differential Geometry 20 (1984), 55-119.

[M2] __, Manifolds with cusps of rank one: spectral theory and $L_{2}$-index theorem, Lecture Notes in Math., no. 1244, Springer-Verlag, New York, 1987.

[Q] D. Quillen, Superconnections and the Chern character, Topology 24 (1985), 89-95.

[S] M. Stern, $L^{2}$-index theorems on locally symmetric spaces (preprint).

[W] E. Witten, Global gravitational anomalies, Comm. Math. Phys. 100 (1985), 197-229.

Département de Mathématiques, Université de Paris Sud, 91405 Orsay, France

Department of Mathematics, State University of New York at Stony Brook, Stony BROOK, NEW YORK 11794 